\title{
Solving the Eikonal equation for compressional and shear waves in anisotropic media using peridynamic differential operator
}

\author{
Ali Can Bekar ${ }^{a}$, Erdogan Madenci ${ }^{\text {a,1 }}$, Ehsan Haghighat ${ }^{\text {b,c }}$, \\ Umair bin Waheed $^{\mathrm{d}}$, Tariq Alkhalifah ${ }^{\mathrm{e}}$ \\ ${ }^{a}$ Department of Aerospace and Mechanical Engineering, The University of Arizona, Tucson, AZ 85721, USA \\ ${ }^{\mathrm{b}}$ Department of Civil and Environmental Engineering, Massachusetts Institute of Technology, Cambridge, MA 02139, \\ USA \\ ${ }^{\mathrm{c}}$ Department of Civil Engineering, University of British Columbia, Vancouver, BC V6T 1Z4, Canada \\ ${ }^{\mathrm{d} D e p a r t m e n t ~ o f ~ G e o s c i e n c e s, ~ K i n g ~ F a h d ~ U n i v e r s i t y ~ o f ~ P e t r o l e u m ~ a n d ~ M i n e r a l s, ~ D h a h r a n ~ 31261, ~ S a u d i ~ A r a b i a . ~}$ \\ ${ }^{\mathrm{e}}$ Physical Sciences and Engineering Division, King Abdullah University of Science and Technology, Thuwal 23955, \\ Saudi Arabia.
}

\section{SUMMARY}

Traveltimes of compressional $(\mathrm{P})$ and shear $(\mathrm{S})$ waves have proven essential in many earthquake and exploration seismology applications. An accurate and efficient traveltime computation for $\mathrm{P}$ and $\mathrm{S}$ waves is crucial for the success of these applications. However, solving the Eikonal equation with a complex phase velocity field in anisotropic media is chállenging. The Eikonal equation is a first-order nonlinear hyperbolic partial differential equation (PDE). It represents the high-frequency asymptotic approximation of the wave equation. The fast marching and sweeping methods are commonly used due to their efficiency in numerically solving the Eikonal equation. However, these methods suffer from numerical inaccuracy in anisotropic media with sharp heterogeneity, irregular surface topography, and complex phase velocity fields. This study presents a new method for the solution of the Eikonal equation by employing the peridynamic differential operator (PDDO). The PDDO provides the nonlocal form of the Eikonal equation by introducing an internal length parameter (horizon) and a weight function with directional nonlocality. The operator is immune to discontinuities in the form of sharp changes in field or model variables and invokes the direction of traveltime in a consistent manner. The weight function controls the strength of association among points within the horizon. Solutions are constructed in a consistent manner without upwind assumptions through simple discretization. The robustness of this approach is established by considering different types of Eikonal equations on complex velocity models in anisotropic media. The examples demonstrate its unconditional numerical stability and results compare well with the reference solutions.

Key words: Anisotropy; seismic modeling; Eikonal equation; Peridynamic differential operator

${ }^{1}$ Corresponding author. Tel.: +1 5206216113 .

E-mail addresses: acbekar@email.arizona.edu (A. C. Bekar), madenci@email.arizona.edu (E. Madenci), ehsanh@mit.edu (E. Haghighat), umair.waheed@kfupm.edu.sa, (U. b. Waheed), tariq.alkhalifah@kaust.edu.sa (T. Alkhalifáh) 


\section{INTRODUCTION}

The Eikonal equation is a nonlinear, first-order, hyperbolic partial differential equation (PDE). It represents a high-frequency asymptotic approximation of the wave equation. Despite its origin in optics, the Eikonal equation appears in modeling many applications across science and engineering disciplines. In image processing, it is used for distance field computations (Adalsteinsson \& Sethian 1995), image denoising (Malladi \& Sethian 1996), segmentation (Alvino et al. 2007), and registration (Cao et al. 2004). It is also used to compute geodesic distances in computer graphics for obtaining the shortest paths on discrete and parametric surfaces (Raviv et al. 2011). In robotics, it finds applications for path planning and navigation (Garrido et al. 2016). In the semiconductor industry, the Eikonal equation is used for simulating various manufacturing processes (Helmsen et al. 1996). Moreover, in seismølogy, it is commonly used to perform traveltime tomography (Taillandier et al. 2009), microseismic source localization (Grechka et al. 2015), and Kirchhoff migration (Lambare et al. 2003), etc.

Traditionally, ray-tracing and finite difference methods have been the two most commonly used approaches for solving the Eikonal equation. In the ray tracing approach, a set of linear ordinary differential equations (ODEs), which represents the characteristic solutions of the Eikonal equation, is often employed. The solution to these ODEs offers the parametric description of the propagation of wave energy inside the computational domain as a function of time (Bullen 1937). Although the ray tracing method is quite efficient, it exhibits several limitations for practical applications, such as the need for traveltime interpolation onto a regular grid for imaging of tomography applications, which becomes difficult when rays diverge due to inhomogeneity in the medium; thus, leading to large spatial gaps. Also, multivalued traveltimes 
arising from the presence of triplications result in uncertainty in the phase computation. Hence, it becomes challenging to find the path with the minimum traveltime.

In view of these challenges, several techniques were introduced over the years for the numerical solution of the Eikonal equation (Vidale 1989; Ventura \& Ahmad 2015; Sethian 1996). The fast marching method (FMM) and the fast sweeping method (FSM) have emerged as the most commonly accepted techniques due to their efficiency and stability properties. The FMM belongs to the family of algorithms, referred to as the single-pass methods. It has links to Dijkstra's algorithm for the traveling salesman problem (Dijkstra 1959). Dijkstra-like shortest path method is combined with an upwind finite difference scheme for solving the Eikonal equation/(Tsitsiklis 1995), which is extended in a level-set context (Sethian 1996). The main idea of FMM is to track a wavefront evolving in the direction of its normal with a given propagation speed. Unlike the FMM, FSM is a multi-pass algorithm. It employs the Gauss-Seidel method along with alternating sweeping direction to solve the Eikonal equation (Zhao 2005). It divides the characteristics belonging to the Eikonal equation into segments and the information flowing along each segment is accounted for by one of the sweeping directions. Both FMM and FSM were initially developed to solve the Eikonal equation for a medium with an isotropic velocity field. However, the isotropic velocity field is an idealization of the subsurface; the Earth's interior is anisotropic in nature.

Therefore, both FMM and FSM were considered extensively to address anisotropic velocity fields (Grechka et al. 2015; Qin et al. 1992; Luo \& Qian 2012) during the last decade. Also, these methods received considerable research interest to attain improvements in the solution accuracy (Popovici \& Sethian 2002), incorporate surface topography (Lan \& Zhang 2013), and utilize parallel implementation for speed ups in the computation (Zhang \& Qian 2004; Detrixhe \& Gibou 
2016). While these advancements have considerably improved the capabilities of FMM and FSM, they also introduce considerable complexity to the original algorithm by requiring higher-order approximation of the derivatives, particularly in the presence of sharp heterogeneities along with irregular surface topography. Moreover, it is well known that the computational cost of FSM or FMM increases dramatically for anisotropic media compared to the isotropic case.

Recently, there is increased attention to describing the response of a system through nonlocal integro-differential operators (Silling 2000; Eringen 2002). A recent class of numerical methods, known as Peridynamic Differential Operator (PDDO) (Madenci et al. 2016; Madenci et al. 2017; Madenci et al. 2019), offers a unified approach to solve both local and nonlocal differential equations of arbitrary order using Taylor series expansion. This approach does not require a uniform discretization and is inherently suitable for solving problems with strong heterogeneity. Therefore, it is particularly suitable for geophysical problems where sharp heterogeneities are often the reason for the deficiency encountered with conventional numerical solvers. The PDDO is free of any spatial derivatives. Therefore, it is immune to discontinuities in the field and model variables, and it provides the ability to embed the information in the direction in which information travels in a consistent manner. It converts the differentiation to numerical integration; thus, the governing equations are always valid throughout the domain. In other words, the derivatives are evaluated based on the interaction strength between neighboring discretization points. The degree of interaction among these points is controlled by a weight function over the active interaction domain, known as the horizon. Also, it invokes directional nonlocality based on the knowledge of characteristic directions along which information travels. The solution is achieved through simple discretization without any special treatments. The PDDO provides accurate solutions to a variety of partial differential equations and serves as a data assimilation 
tool for denoising and data recovery (Madenci et al. 2017; Madenci et al. 2019). In a recent study, the PDDO has been applied to the solution of rather challenging nonlinear PDEs such as Burgers, Swift-Hohenberg, Korteweg-de Vries, Kuramoto-Sivashinsky, nonlinear Schrödinger, and CahnHilliard equations (Bekar \& Madenci 2021).

In order to remove the difficulties faced with FMM and FSM, this study presents a new computational approach to solve the Eikonal equation by employing the PDDO. The PDDQ provides the nonlocal form of the Eikonal equation by introducing an internal length parameter (horizon) and a weight function with directional nonlocality. Therefore, it is extremely suitable for solving the Eikonal equation for traveltimes due to compressional (P) and shear (S) waves in sharply heterogeneous velocity fields and in the presence of sharp variations in surface topography in an anisotropic media. Unlike the FSM and FMM, the PDDO solution does not rely on the information from the neighboring grid points. Therefore, it is much easier to parallelize the solver for improvement in its computational efficiency. Furthermore, numerical stability is always ensured and solutions compare well with the reference solutions.

In the next section, we present different forms of the Eikonal equation applicable to seismology studies based on travel time computations. Subsequently, we explain the peridynamic differential operator (PDDO) sølution method with a brief discussion on its numerical implementation. Finally, we demonstrate the accuracy of the PD solutions by using 1) the isotropic constant gradient model, 2) the isotropic Marmousi model, 3) the BP TTI model, and 4) the qSV-wave Eikonal equations previously considered by others using FMM and FSM. Furthermore, we provide additional solutions to the elliptically isotropic Marmousi model and the Hess VTI model as presented in the appendix to expose the generality of the PDDO. We demonstrate that these solutions are more accurate than the FSM results. While this is the first 
study in the use of nonlocal PDDO for solving Eikonal equations, it builds the foundation for further studies on the nonlocal considerations in earthquake travel time computations.

\section{THE EIKONAL EQUATION}

The eikonal equation is a time independent first order nonlinear hyperbolic PDE. In geophysics, it describes the traveltime, $T=T(x, z)$, of propagating waves in a $2 \mathrm{D}$ medium bounded by $x \in\left[L_{1}, L_{2}\right]$ and $z \in\left[W_{1}, W_{2}\right]$. It is subjected to a constraint at the source location given by $T\left(x_{s}, z_{s}\right)=0$. The coordinates $x$ and $z$ represent the offset and depth from the source in the domain of interest, respectively. The traveltime depends on the source location, anisotropy and the type of wave propagation.

The Eikonal equation for an inhomogeneous anisotropic viscoelastic medium can be derived from the Christoffel equation (Musgrave 1970; Sharma 2008)

$\Gamma_{i k}(\mathbf{x}, \mathbf{p})=a_{i j k l} p_{j} p_{l}-\delta_{i k}$ for $i, j, k, l=x, y, z$

in which $a_{i j k l}$ is the density normalized viscoelastic material property tensor (Vavrycuk 2009). It can be expressed as

$$
a_{i j k l}=a_{i j k l}^{R}+i a_{i j k l}^{I}
$$

where the imaginary part represents the attenuation effects. However, it is omitted under an elastic medium assumption leading to

$$
a_{i j k l}=a_{i j k l}^{R}=C_{i j k l} / \rho
$$

in which $C_{i j k l}$ is the material property tensor. In Voigt notation for an orthotropic medium, it can be expressed as 


$$
C=\left[\begin{array}{cccccc}
C_{11} & C_{12} & C_{13} & 0 & 0 & 0 \\
C_{12} & C_{22} & C_{23} & 0 & 0 & 0 \\
C_{13} & C_{23} & C_{33} & 0 & 0 & 0 \\
0 & 0 & 0 & C_{44} & 0 & 0 \\
0 & 0 & 0 & 0 & C_{55} & 0 \\
0 & 0 & 0 & 0 & 0 & C_{66}
\end{array}\right]
$$

Prior to solving for the characteristic equation of the Christoffel matrix, the following nine parameters are introduced as (Alkhalifah 2003):

1. P-wave vertical velocity

$v_{v}=\sqrt{\frac{C_{33}}{\rho}}$

2. Vertical velocity of S-wave polarized along $x_{1}$-direction

$$
v_{s 1}=\sqrt{\frac{C_{55}}{\rho}}
$$

3. Vertical velocity of $\mathrm{S}$-wave polarized along $x_{2}$-direction

$$
v_{s 2}=\sqrt{\frac{C_{44}}{\rho}}
$$

4. Horizontal velocity of S-wave polarized along $x_{2}$-direction

$$
v_{s 3}=\sqrt{\frac{C_{66}}{\rho}}
$$

\footnotetext{
5. Normal moveout $\mathrm{P}$-wave velocity for horizontal reflectors in the $\left(x_{1}-x_{3}\right)$ plane
} 
$v_{1}=\sqrt{\frac{C_{13}\left(C_{13}+2 C_{55}\right)+C_{33} C_{55}}{\rho\left(C_{33}-C_{55}\right)}}$

6. Normal moveout P-wave velocity for horizontal reflectors in the $\left(x_{2}-x_{3}\right)$ plane

$v_{2}=\sqrt{\frac{C_{23}\left(C_{23}+2 C_{44}\right)+C_{33} C_{44}}{\rho\left(C_{33}-C_{44}\right)}}$

7. Parameter in the $\left(x_{1}-x_{3}\right)$ symmetry plane

$\eta_{1}=\frac{C_{11}\left(C_{33}-C_{55}\right)}{2 C_{13}\left(C_{13}+2 C_{55}\right)+2 C_{33} C_{55}}-\frac{1}{2}$

8. Parameter in the $\left(x_{2}-x_{3}\right)$ symmetry plane

$\eta_{2}=\frac{C_{22}\left(C_{33}-C_{44}\right)}{2 C_{23}\left(C_{23}+2 C_{44}\right)+2 C_{33} C_{44}}-\frac{1}{2}$

9. Parameter in the $\left(x_{1}-x_{2}\right)$ plane with respect to $x_{1}$-direction

$\delta=\frac{\left(C_{12}+C_{66}\right)^{2}-\left(C_{11}-C_{66}\right)^{2}}{2 C_{11}\left(C_{11}-C_{66}\right)}$

For a transversély isotropic and orthotropic media, setting qS-wave velocity to zero along the symmetry axis does not affect the accuracy of qP-wave traveltime calculation (Alkhalifah 1998; Waheed et al. 2015). Therefore, the solution to the characteristic equation of the Christoffel matrix with zero values of $v_{s 1}, v_{s 2}$ and $v_{s 3}$ leads to the expression for $p_{z}$ as 
$p_{, z}^{2}=\frac{1}{v_{v}^{2}}\left(1-\frac{v_{1}^{2} p_{, x}^{2}}{1-2 \eta_{1} v_{1}^{2} p_{, x}^{2}}\right)$

The variable after subscript comma denotes differentiation with respect to that variable. After defining $v=v_{1}=v_{v}$ as the phase velocity, $\eta=\eta_{1}=\eta_{2}$ and $\varepsilon=\eta(1+2 \delta)+\delta$ as anisotropy parameters. this equation can be recast as

$(1+2 \varepsilon) p_{, x}^{2}+p_{, z}^{2}\left(1-R p_{, x}^{2}\right)=\frac{1}{v^{2}}$

It is referred to as the Eikonal equation for $\mathrm{P}$ waves in a medium with tilted transverse isotropy (TTI) (Alkhalifah 1998; Alkhalifah 2000; Waheed \& Alkhalifah 2017; Waheed et al. 2021). The known medium coefficient, $R(x, z)$ is defined as

$R=\frac{2 \eta v^{2}(1+2 \varepsilon)}{1+2 \eta}$

The horizontal and vertical slowness components, $p_{x}$ and $p_{z}$, respectively, are defined as

$p_{x}=T_{, x} \cos \theta+T_{, z} \sin \theta$

and

$p_{z}=T_{, z} \cos \theta-T_{, x} \sin \theta$

where $\theta=\theta(x, z)$ is the symmetry axis angle.

In a medium with vertical transverse isotropy (VTI) for $\theta=0$, the Eikonal equation reduces to 


$$
(1+2 \varepsilon) T_{, x}^{2}+T_{, z}^{2}\left(1-R T_{, x}^{2}\right)=\frac{1}{v^{2}}
$$

This equation can be further simplified to its elliptically isotropic form by setting $\eta=0$ as

$$
(1+2 \varepsilon) T_{, x}^{2}+T_{, z}^{2}=\frac{1}{v^{2}}
$$

Furthermore, with $\varepsilon=0$, it reduces to its simplest form for an isotropic medium as

$$
T_{, x}^{2}+T_{, z}^{2}=\frac{1}{v^{2}}
$$

In a medium with TTI, the coupled $\mathrm{qP}$ and $\mathrm{qSV}$ waves form a quartic surface of slowness given by Han et al. (2017)

$$
p_{z}^{4}-B p_{z}^{2}+C=0
$$

where

$$
B=\frac{1}{v_{p}^{2}}+\frac{1}{v_{s}^{2}}-2\left(1+\delta+(\epsilon-\delta) \frac{v_{p}^{2}}{v_{s}^{2}}\right) p_{x}^{2}
$$


$C=\left((1+2 \epsilon) p_{x}^{2}-\frac{1}{v_{p}^{2}}\right)\left(p_{x}^{2}-\frac{1}{v_{s}^{2}}\right)$

in which $v_{p}=v_{p}(x, z)$ and $v_{s}=v_{s}(x, z)$ represent the phase velocity of the medium in $\mathrm{P}$ and $\mathrm{S}$ waves in the vertical direction, respectively, and $\epsilon=\epsilon(x, z)$ and $\delta=\delta(x, z)$ are referred to as Thomsen parameters. The roots of Eq. (22) represent the qSV-and qP-wave slowness surfaces. The qP-wave travels faster than the qSV-wave; thus, leading to

$p_{z}^{2}-\frac{B+\sqrt{B^{2}-4 C}}{2}=0$ for qSV-wave

and

$p_{z}^{2}-\frac{B-\sqrt{B^{2}-4 C}}{2}=0$ for $\mathrm{qP}$-wave.

\section{PERIDYNAMIC DIFFERENTIAL OPERATOR}

Madenci et al. (2016, 2017, 2019) introduced the Peridynamic Differential Operator (PDDO) to construct the nonlocal representation of a scalar field $f=f(\mathbf{x})$ and its derivatives at point $\mathbf{x}$ by considering its interactions with the other points, $\mathbf{x}^{\prime}$, in its interaction domain known as horizon, as shown in red in Fig. 1. It provides differentiation of $N$-th order in $M$ dimensions through integration without a medium smoothness requirement. 


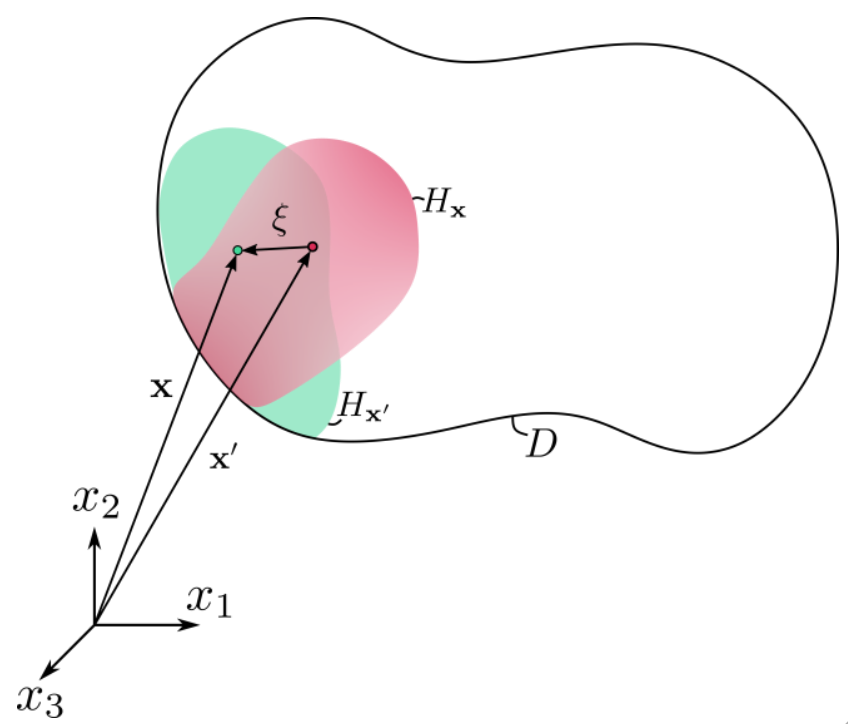

Figure 1. The Peridynamic interaction domains, a.k.a. horizons, red and green, with arbitrary shape and size, for the discretized points $\mathbf{x}$ and $\mathbf{x}^{\prime}$, respectively (Madenci et al. 2019).

The derivation of PDDO utilizes the concept of PD interactions (Madenci et al. 2016; Madenci et al. 2019) and the construction of PD functions that are orthogonal to each term in the Taylor Series Expansion (TSE). In the discretized domain, each point has its own family members. The points $\mathbf{x}$ and $\mathbf{x}^{\prime}$ only interact with the other points in their own interaction domains, $H_{\mathbf{x}}$ and $H_{\mathbf{x}^{\prime}}$, respectively. The relative position vector between these points is defined as $\xi=\mathbf{x}^{\prime}-\mathbf{x}$. The interaction domains for points $\mathbf{x}$ and $\mathbf{x}^{\prime}$ do not need to be symmetric, which makes PDDO an attractive method for a variety of problems.

Based on the PDDO, the $N$-th order derivative of a function $f(\mathbf{x})$ with $M$ dimensions is expressed as (Madenci et al. 2016; Madenci et al. 2019) 
$\frac{\partial^{r_{1}+r_{2}+\cdots+r_{N}} f(\mathbf{x})}{\partial x_{1}^{r_{1}} \partial x_{2}^{r_{2}} \cdots \partial x_{M}^{r_{N}}}=\int_{H_{\mathbf{x}}} f(\mathbf{x}+\xi) g_{N}^{r_{1} r_{2} \cdots r_{N}}(\boldsymbol{\xi}) d \xi_{1} d \xi_{2} \cdots d \xi_{M}$

in which $r_{i}$ denotes the order of differentiation with respect to variable $x_{i}$ with $i=1, \ldots ., M$, and $g_{N}^{r_{1} r_{2} \cdots r_{N}}(\xi)$ are the PD functions (see Madenci et al. (2019) for further details).

Since only the first order derivatives of traveltime, $T(x, z)$ appear in the Eikonal equation, its TSE is expressed as

$T(\mathbf{x}+\xi)=T(\mathbf{x})+\xi_{x} \frac{\partial T(\mathbf{x})}{\partial x}+\xi_{z} \frac{\partial T(\mathbf{x})}{\partial z}+R_{1}(\mathbf{x})$

where $\mathbf{x}^{T}=\{x, z\}, \xi_{x}$ and $\xi_{z}$ are the components of the vector $\xi$ and $R_{1}(\mathbf{x})$ represents the remainder of the $1^{\text {st }}$ order approximation. Multiplying each term with PD functions, $g_{1}^{p_{1} p_{2}}(\xi)$ and integrating over the domain of interaction (family), $H_{\mathrm{x}}$ result in

$$
\begin{aligned}
\int_{H_{\mathbf{x}}} T(\mathbf{x}+\xi) g_{1}^{r_{x} r_{z}}(\xi) d V= & T(\mathbf{x}) \int_{H_{\mathbf{x}}} g_{1}^{r_{x} r_{z}}(\xi) d H_{\mathbf{x}^{\prime}}+ \\
& \frac{\partial T(\mathbf{x})}{\partial x} \int_{H_{\mathbf{x}}} \xi_{x} g_{1}^{r_{1} r_{z}}(\xi) d H_{\mathbf{x}^{\prime}}+\frac{\partial T(\mathbf{x})}{\partial z} \int_{H_{\mathbf{x}}} \xi_{z} g_{1}^{r_{x} r_{z}}(\xi) d H_{\mathbf{x}^{\prime}}
\end{aligned} .
$$

The PD functions must be orthogonal to each term in the TSE as 
with $\left(s_{x}, s_{z}, r_{x}, r_{z}=0,1\right)$ and $\delta_{s_{i} r_{j}}$ as the Kronecker delta symbol. Applying the orthogonality conditions, Eq. (30) results in PD form of the function itself and its first order derivatives as

$$
T^{P D}(\mathbf{x})=\int_{H_{\mathbf{x}}} T(\mathbf{x}+\xi) g_{1}^{00}(\xi) d H_{\mathbf{x}^{\prime}}
$$

and

$$
\left\{\begin{array}{l}
T_{, x}^{P D} \\
T_{, z}^{P D}
\end{array}\right\}=\int_{H_{\mathbf{x}}} T(\mathbf{x}+\xi)\left\{\begin{array}{l}
g_{1}^{10}(\xi) \\
g_{1}^{01}(\xi)
\end{array}\right\} d H_{\mathbf{x}^{\prime}} .
$$

As detailed in Madenci et al. (2019), the 1-st order PD functions are constructed using linear polynomial basis functions as

$$
g_{1}^{r_{x} r_{z}}(\xi)=a_{00}^{r_{x} r_{z}} w(|\xi|)+a_{x 0}^{r_{x} r_{z}} w(|\xi|) \xi_{x}+a_{0 z}^{r_{x} r_{z}} w(|\xi|) \xi_{z}
$$

where $a_{s_{x} s_{z}}^{r_{x} r_{z}}$ are the unknown coefficients and $w(|\xi|)$ is the non-dimensional weight function which controls the strength of interactions among the family members. It may vary from point to point and can also be modified to invoke directional nonlocality based on the direction of information travel. Substituting the PD functions back into the orthogonality equation, Eq. (30) after algebraic manípulations leads to a system of equations that determines the coefficients $a_{s_{x} s_{z}}^{r_{x} r_{z}}$ as 
$\mathbf{A a}=\mathbf{b}$

in which

$\mathbf{A}=\int_{H_{\mathbf{x}}} w(|\xi|)\left[\begin{array}{ccc}1 & \xi_{x} & \xi_{z} \\ \xi_{x} & \xi_{x}^{2} & \xi_{x} \xi_{z} \\ \xi_{z} & \xi_{x} \xi_{z} & \xi_{z}^{2}\end{array}\right] d H_{\mathbf{x}^{\prime}}$

$\mathbf{a}=\left[\begin{array}{lll}a_{00}^{00} & a_{00}^{10} & a_{00}^{01} \\ a_{x 0}^{00} & a_{x 0}^{10} & a_{x 0}^{01} \\ a_{0 z}^{00} & a_{0 z}^{10} & a_{0 z}^{01}\end{array}\right]$

(36)

and

$\mathbf{b}=\left[\begin{array}{lll}1 & 0 & 0 \\ 0 & 1 & 0 \\ 0 & 0 & 1\end{array}\right]$.

Determination of the coefficients, $a_{s_{x} s_{z}}^{r_{x} r_{z}}$ via $\mathbf{a}=\mathbf{A}^{-1} \mathbf{b}$ establishes the PD functions $g_{1}^{r_{1} r_{z}}(\boldsymbol{\xi})$. In depth description of the derivation and many applications concerning the solutions of linear and nonlinear ordinary and partial differential equations as well as the relevant source codes are given by Madenci et al. (2019). 
The integrals are evaluated through a meshless quadrature technique. Considering a uniform grid spacing of $\Delta$, the size of the horizon can be defined as $\delta=m \Delta$, with $m$ as the number of family points in each direction. To have a stable discretization, $m$ should be selected such that $N \leq m \leq N+2$, with $N=1$ as the highest order spatial derivative appearing in the PDE. For a fixed value of $m$, the PD representation must converge to the local form as the parameter $\delta$ approaches zero. This discretization for a two-dimensional domain is shown in Fig. 2. The interior points can have a symmetric family. However, the boundary points always have a nonsymmetric family.

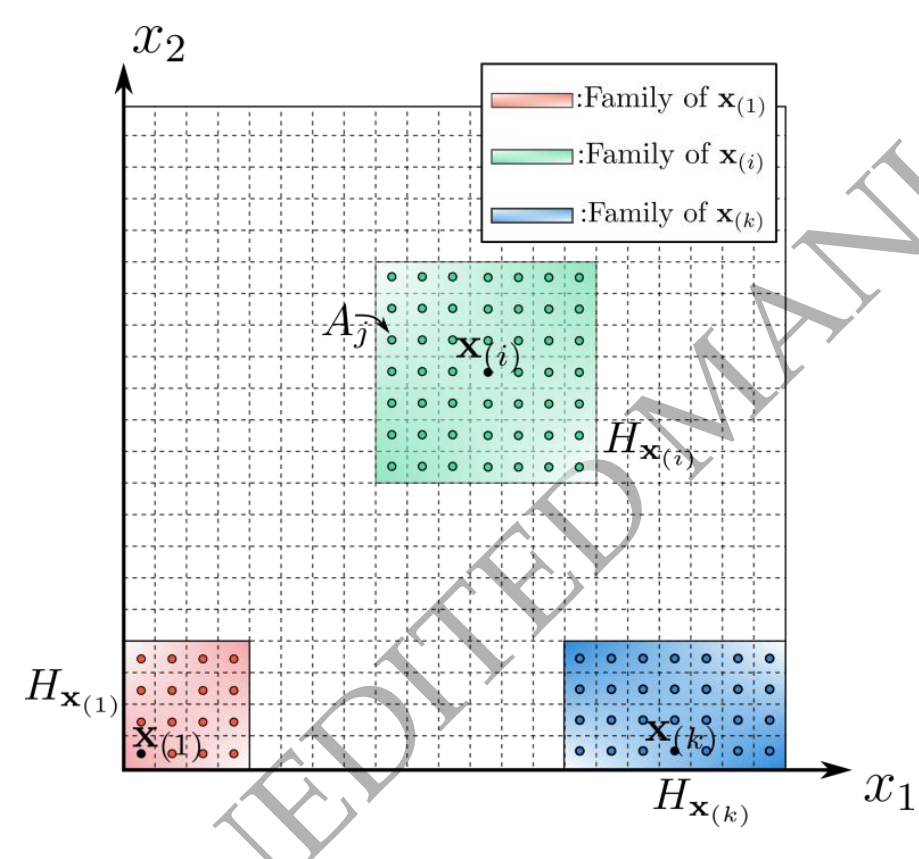

Figure 2. Déscription of families in a PD computational domain

The discretized form of Eqs. (31) and (32) are expressed as

$T^{P D}\left(\mathbf{x}_{(k)}\right)=\sum_{j=1}^{N N_{(k)}} T\left(\mathbf{x}_{(j)}\right) g_{1}^{00}\left(\mathbf{x}_{(j)}-\mathbf{x}_{(k)}\right) A_{(j)}$ for $k=1, \ldots, K$ 
and

$\left\{\begin{array}{l}T_{, x}^{P D}\left(\mathbf{x}_{(k)}\right) \\ T_{, z}^{P D}\left(\mathbf{x}_{(k)}\right)\end{array}\right\}=\sum_{j=1}^{N_{(k)}} T\left(\mathbf{x}_{(j)}\right)\left\{\begin{array}{l}g_{1}^{10}\left(\mathbf{x}_{(j)}-\mathbf{x}_{(k)}\right) \\ g_{1}^{01}\left(\mathbf{x}_{(j)}-\mathbf{x}_{(k)}\right)\end{array}\right\} A_{(j)}$ for $k=1, \ldots, K$

in which $A_{(j)}$ represents the area of each point, $\mathbf{x}_{(j)}$ and $N_{(k)}$ denotes the number of family members of point $\mathbf{x}_{(k)}$. Thus, the summation accounts for all of the interactions of point $\mathbf{x}_{(k)}$ within its family. The Gaussian quadrature rule is employed with unit integration weights. The total number of grid points in the computational domain is denoted by $K$.

For a point symmetrically located a circular interaction domain with horizon, $\delta$, the PD functions have analytical expressions; thus, leading to

$T^{P D}(\mathbf{x})=\frac{1}{2 \pi \delta^{2}} \int_{H_{\mathbf{x}}} w(|\xi|) T(\mathbf{x}+\xi) d A$

and

$$
\left\{\begin{array}{l}
T_{, x}^{P D} \\
T_{, z}^{P D}
\end{array}\right\}=\frac{2}{\pi \delta^{4}} \int_{H_{\mathbf{x}}} w(|\xi|) T(\mathbf{x}+\xi)\left\{\begin{array}{l}
\xi_{x} \\
\xi_{z}
\end{array}\right\} d A
$$

In order to invoke the direction of travel information during the solution, the PD functions are constructed by employing a weight function with directional nonlocality. The weight function is 
updated depending on the position of the point inside the family and the gradient direction of the traveltime at the point of interest, as illustrated in Fig. 3. It is expressed as

$$
w(|\xi|, \kappa ; \delta)=\kappa e^{-4(\mid \xi / \delta)^{2}}
$$

with

$$
\kappa=\left\{\begin{array}{lll}
0.1 & \text { if } & \xi \cdot \nabla T<0 \\
1.0 & \text { if } & \xi \cdot \nabla T \geq 0
\end{array}\right.
$$

in which the horizon, $\delta$ defines the extent of interaction domain, $H_{\mathbf{x}}$ for point $\mathbf{x}$ and the parameter $\kappa$ allows for the information travel with directional nonlocality (upwinding) as illustrated in Fig. 4. It offers a simple way to reflect the effect of upwinding direction and control the strength of interaction among the points while ensuring numerical stability. 


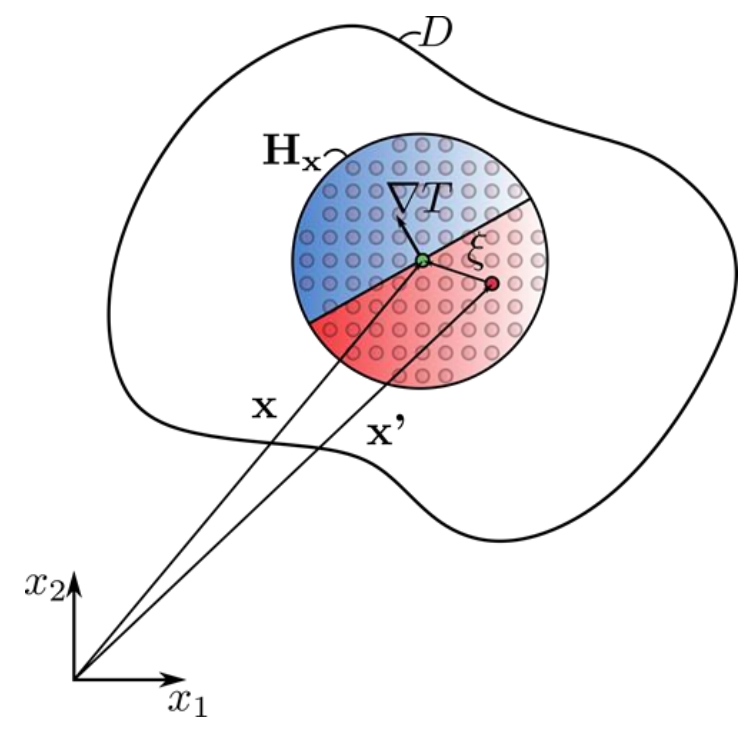

Figure 3. Directional nonlocality in the interaction domain of point $\mathbf{x}$

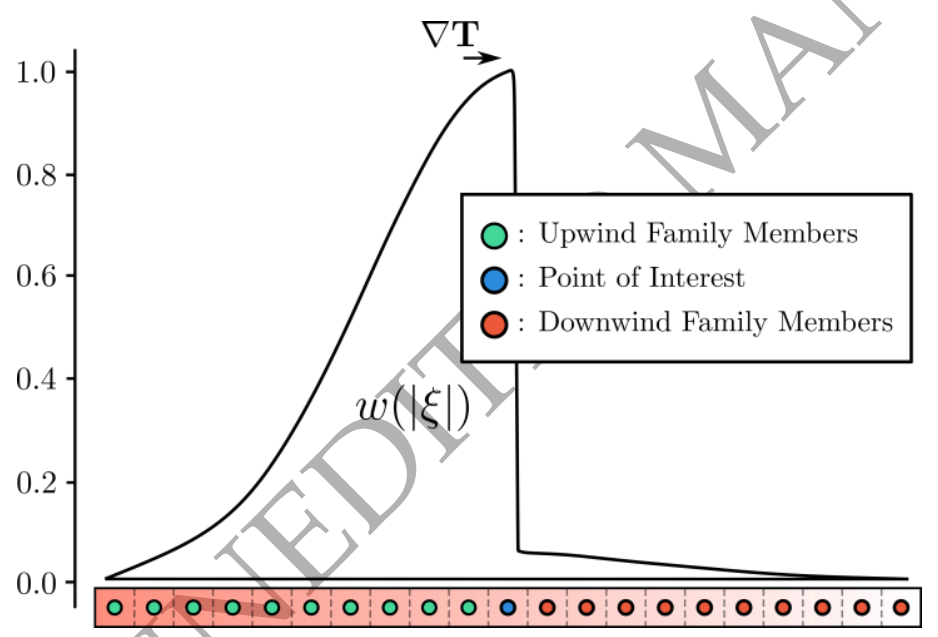

Figure 4. Tailored weight function for directional interaction between points

\section{NUMERICAL IMPLEMENTATION}

Replacing the local derivatives, $T_{, x}$ and $T_{, z}$ in the Eikonal equation with their PD counterparts,

$T_{x}^{P D}$ and $T_{, z}^{P D}$ leads to its discrete form at each PD point in the domain. Similarly, the constraint 
condition $T\left(\mathbf{x}_{s}\right)=0$ is replaced with $T^{P D}\left(\mathbf{x}_{s}\right)$. It is enforced by deleting the corresponding row in the resulting nonlinear algebraic system of equations. The resulting system of equations can be written in the form

$\mathbf{F}(\mathbf{u})=\mathbf{0}$

where the vector $\mathbf{u}$ includes the PD unknowns, $T\left(\mathbf{x}_{(k)}\right)$. These nonlinear equations are solved by Newton-Raphson method in an iterative manner. The incremental residual vector, $\Delta \mathbf{F}$ can be obtained from the TSE arising from the incremental changes in the unknown vector, $\Delta \mathbf{u}$ as

$\mathbf{F}(\mathbf{u}+\Delta \mathbf{u})=\mathbf{F}(\mathbf{u})+\frac{\partial \mathbf{F}}{\partial \mathbf{u}} \Delta \mathbf{u}+R_{1}(\mathbf{u})$.

Disregarding the remainder terms, $R_{1}(\mathbf{u})$ leads to the incremental vector, $\Delta \mathbf{F}$ as

$\Delta \mathbf{F}=\mathbf{F}(\mathbf{u}+\Delta \mathbf{u})-\mathbf{F}(\mathbf{u})=\frac{\partial \mathbf{F}}{\partial \mathbf{u}} \Delta \mathbf{u}$

where $\Delta \mathbf{u}$ is the unknøwn incremental vector and $\mathbf{J}=\frac{\partial \mathbf{F}}{\partial \mathbf{u}}$ is the Jacobian matrix.

In the numérical implementation, satisfying Eq. (44) by using Eq. (46) in one step is not tractable. Thus, the solution is achieved iteratively through a recursive form (fixed point iteration) as 
$\left(\frac{\partial \mathbf{F}}{\partial \mathbf{u}}\right)^{(n)} \Delta \mathbf{u}^{(n+1)}=-\mathbf{F}\left(\mathbf{u}^{n}\right)$

or

$\Delta \mathbf{u}^{(n+1)}=-\mathbf{J}^{-1}\left(\mathbf{u}^{(n)}\right) \mathbf{F}\left(\mathbf{u}^{n}\right)$

with

$\mathbf{J}\left(\mathbf{u}^{(n)}\right)=\frac{\partial \mathbf{F}\left(\mathbf{u}^{(n)}\right)}{\partial \mathbf{u}}$

in which $\mathbf{u}^{(n+1)}=\mathbf{u}^{(n)}+\Delta \mathbf{u}^{(n+1)}$ with $n$ representing the iteration number in the algorithm. The Jacobian matrix is evaluated through automatic differentiation, and the equations are solved by employing the Generalized Minimal RESidual method (GMRES) with ILU preconditioner. The family member construction is achieved by the KD-tree algorithm (Bentley 1975). Depending on the position of family members and the gradient direction of the traveltime, the weight function and the PD functions are updated during each iteration. The convergence of the solution is achieved when a specified tolerance is satisfied, i.e., $\|\mathbf{F}(\mathbf{u})\|<e$.

The initial guess for the iterative method is constructed in a consistent manner by modifying the analytical solution (Fomel et al. 2009) for a constant gradient velocity profile as 
$T_{\text {initial }}(\mathbf{x})=\frac{1}{\sqrt{\left(\bar{g}_{x}^{2}+\bar{g}_{z}^{2}\right)}} \cosh ^{-1}\left(1+\frac{\left(\bar{g}_{x}^{2}+\bar{g}_{z}^{2}\right)\left|\mathbf{x}-\mathbf{x}_{s}\right|^{2}}{2 \bar{v}\left(\mathbf{x}_{s}\right) \bar{v}(\mathbf{x})}\right)$

where $\bar{v}(\mathbf{x})$ represents the velocity field with approximate constant gradients, $\bar{g}_{x}$ and $\bar{g}_{z}$ in $x$ and $z$-directions, respectively. They are approximated as

$\bar{v}(\mathbf{x})=\bar{v}\left(x=L_{1}, z=W_{1}\right)+\bar{g}_{x} x+\bar{g}_{z} z$

in which

$\bar{g}_{x} \approx \frac{v\left(x=L_{2}, z=\left(W_{2}-W_{1}\right) / 2\right)-v\left(x=L_{1}, z=\left(W_{2}-W_{1}\right) / 2\right)}{L_{2}-L_{1}}$

and

$\bar{g}_{z} \approx \frac{v\left(x=\left(L_{2}-L_{1}\right) / 2, z=W_{2}\right)-v\left(x=\left(L_{2}-L_{1}\right) / 2, z=W_{1}\right)}{W_{2}-W_{1}}$

If the velocity field is coarse, this initial case is used after smoothing by using a Gaussian filter.

With the initial guess from Eq. (50), the solution to the smoothed field is used as an initial guess for the coarse velocity field.

The boundedness of the solution $\mathbf{u}$ depends on the behavior of $-\mathbf{J}^{-1}$. Therefore, the method is stable if the real parts of all eigenvalues of $-\mathbf{J}^{-1}$ are negative. Otherwise, it is unstable. It is worth noting that the real parts of the eigenvalues of $-\mathbf{J}^{-1}$ and $-\mathbf{J}$ have the same signs. Therefore, examining the eigen spectrum of $-\mathbf{J}$ is sufficient. Fig. 5 shows the real and imaginary parts of 
the eigenvalues of $\mathbf{- J}$ corresponding to the weight function with and without directional nonlocality. The Jacobian, $-\mathbf{J}$ corresponds to the $\% 2$ randomly perturbed analytical solution of the isotropic Eikonal equation for the velocity field, $v(\mathbf{x})$ with constant gradients $g_{x}=0$ and $g_{z}=0.5 s^{-1}$ of the form (Fomel et al. 2009)

$T(\mathbf{x})=2 \cosh ^{-1}\left(1+\frac{|\mathbf{x}-(1.0,1.0)|^{2}}{20 v(\mathbf{x})}\right)$

in which the velocity at source $v(1.0,1.0)=2.5 \mathrm{~km} / \mathrm{s}$. The weight function with directional nonlocality results in eigenvalues with only negative real parts. However, the symmetric weight function without directional nonlocality results in eigenvalues with both positive and negative real parts. This ascertains the stability and necessity of modifying the symmetric weight function using the gradient of the travel times. Also, the weight function with directional nonlocality ensures the numerical stability of the solution procedure. 


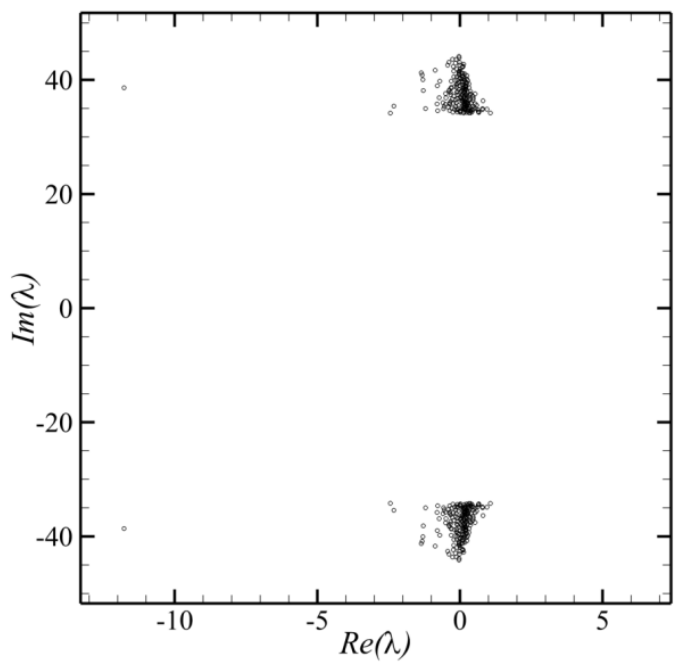

a)

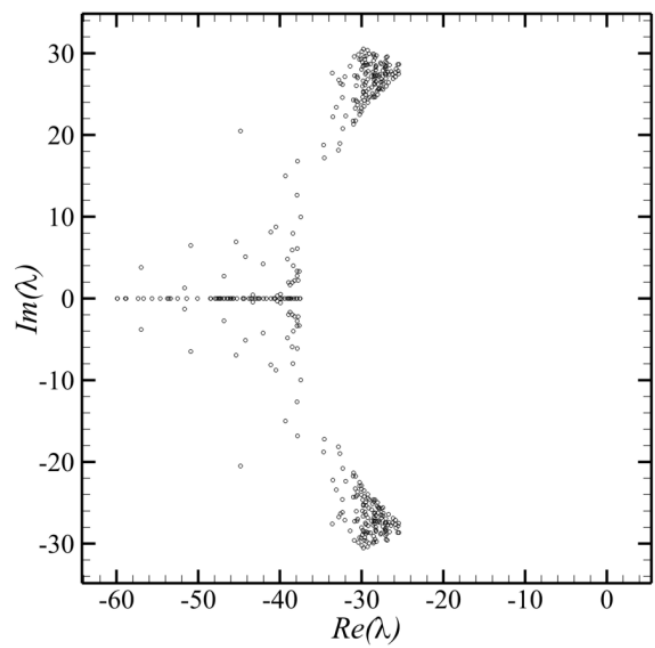

b)

Figure 5. Real and imaginary parts of the eigenvalues of $-\mathbf{J}$ corresponding weight function: a) without directional nonlocality (symmetric), and b) with directional nonlocality.

One of the major advantages of this approach is that it can be applied to media with different types of anisotropy without any special treatments. Even though the gradient of traveltime and the characteristic directions do not coincide in anisotropic media for $\mathrm{qS}$-wave calculations, only the weight functions are adjusted by using the traveltime gradient. This makes the approach relatively straightforward; slight modifications in the software for isotropic media are sufficient to determine the solution in anisotropic media. Also, it is more accurate than conventional methods (FSM and FMM) because the PDDO includes nonlocal interactions between material points. This feature is significant when high gradients or singularities are present in the domain. Furthermore, it is suitable for parallelization since the discrete form of the equations results in a system of algebraic equations. The GPU architecture can accelerate the computations significantly (Boys et al. 2021). 
Its major disadvantage is the requirement of an initial guess for the solution. For a problem with a single source, an approximate initial guess is straightforward; however, it may be difficult for a domain with multiple sources. Another disadvatange is the higher computational cost because of the system of equations that should be solved at each iteration. However, given the increased accuracy in solution and potential for accounting for nonlocality and sharp gradients, the increased computation cost is justifiable.

\section{NUMERICAL RESULTS}

In order to establish the robustness and simplicity of the present approach, the numerical results concern traveltime solutions due to both compressional (qP) and shear (qSV) waves. The PD solutions are obtained by applying the same procedure and discretization parameters discussed above without any special treatment. The horizon size is specified as $\delta=2 \Delta$ and the initial guess is constructed in a consistent manner. The PD qP traveltime solutions are constructed for the isotropic constant gradient, isotropic Marmousi, and BP TTI models due to the availability of FSM solutions for comparison. In order to expose the generality of the PDDO while retaining coherence, additional solutions to the efliptically isotropic Marmousi model and the Hess VTI model are presented in the appendix. For a meaningful comparison, the PD discretization is set to the same grid spacing as that of FSM. As shown in the subsequent sections, the PD predictions are closer to the reference solution (second-order FSM with fine mesh) than those of FSM. Without any special treatment, the PD qSV solution is also readily constructed in an anisotropic medium. However, the predictions are compared with the wave field extrapolation using the lowrank approximation. The PD predictions recover the low-rank solution wave front corresponding 
to the main branch of the wave field. The same procedure applies to the solution of qP traveltime using Eq. (26).

The convergence of the solution is achieved when a specified tolerance is satisfied, i.e., $\|\mathbf{F}(\mathbf{u})\|<e$ while considering a maximum number of iterations and an extra parameter for early stopping. If the error norm does not decrease significantly and admits oscillations within the specified number for early stopping, the procedure is terminated and convergence is achieved. All errors are computed through early stopping in order to achieve the minimum possible error. All of the computations are performed on a Laptop with Intel i7-6700HQ CPU 3.6 GHz frequency and $6 \mathrm{MB}$ cache and 16GBs of DDR4 RAM. The initial guess is also an important factor in the computation time. Therefore, it may take 2 or 3 times more to obtain the solution for a coarse velocity field than the smoothed field because the initial guess is obtained using an analytic solution. The computational time can be reduced by parallelization and GPU architecture which accelerates the PD solver 1.4-10 times faster (Boys et al. 2021),

\subsection{Isotropic Eikonal equation}

\subsubsection{Constant velocity gradient}

An isotropic domain with constant velocity gradients is first considered in order to establish the fidelity of PD predictions by comparison with the analytical solution to the Eikonal equation. The analytical solution is of the form (Fomel et al. 2009)

$$
T(\mathbf{x})=\frac{1}{\sqrt{\left(g_{z}^{2}+g_{z}^{2}\right)}} \cosh ^{-1}\left(1+\frac{\left(g_{x}^{2}+g_{z}^{2}\right)\left|\mathbf{x}-\mathbf{x}_{s}\right|^{2}}{2 v\left(\mathbf{x}_{s}\right) v(\mathbf{x})}\right)
$$


in which $g_{x}$ and $g_{z}$ represent the constant gradients in $x$ - and $z$-directions, respectively, of the velocity field, $v(\mathbf{x})$ with its value, $v\left(\mathbf{x}_{s}\right)$ at source. The velocity field with constant gradients of $g_{x}=0$ and $g_{z}=0.5 s^{-1}$ is shown in Fig. 6 . The source is located at $\mathbf{x}_{s}=(1.0 \mathrm{~km}, 1.0 \mathrm{~km})$ in the domain specified by $x \in[0,2.0 \mathrm{~km}]$ and $z \in[0,2.0 \mathrm{~km}]$. The value of the velocity at source is $v\left(\mathbf{x}_{s}\right)=2.5 \mathrm{~km} / \mathrm{s}$

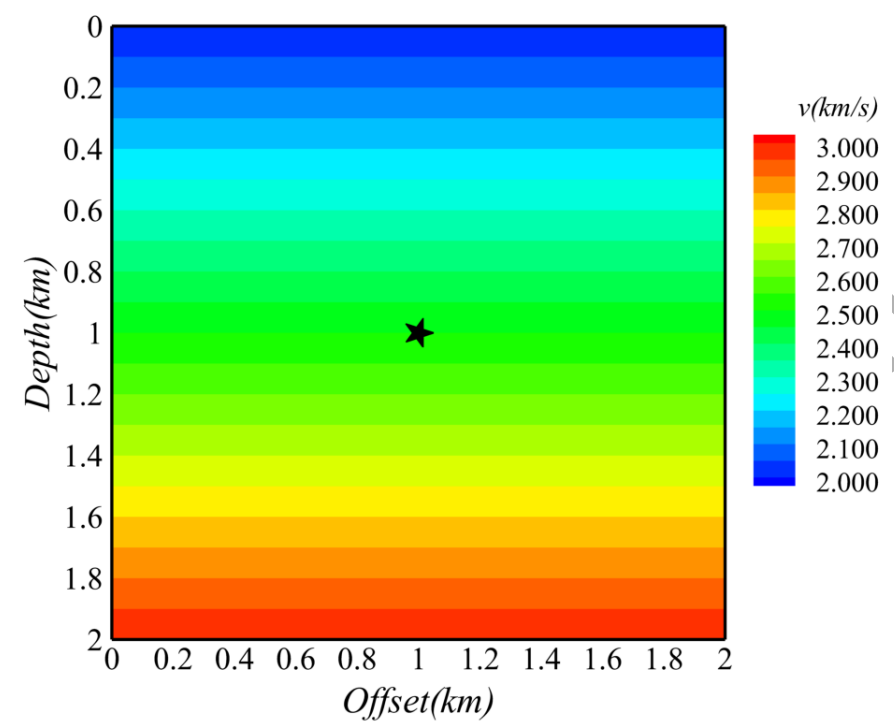

Figure 6. Constant velocity gradient field in the domain with a source at the center

For this velocity field, the analytical solution given by Eq. (55) reduces to

$$
T(\mathbf{x})=2 \cosh ^{-1}\left(1+\frac{|\mathbf{x}-(1.0,1.0)|^{2}}{20 \nu(\mathbf{x})}\right)
$$

Substituting for the local derivatives from Eq. (39) in Eq. (21) results in the PD form of the isotropic Eikonal equation as 


$$
\left(\sum_{j=1}^{N_{(k)}} T_{(j)} g_{1}^{10}\left(\xi_{1(k)(j)}, \xi_{2(k)(j)}\right) A_{(j)}\right)^{2}+\left(\sum_{j=1}^{N_{(k)}} T_{(j)} g_{1}^{01}\left(\xi_{1(k)(j)}, \xi_{2(k)(j)}\right) A_{(j)}\right)^{2}=\frac{1}{v^{2}\left(x_{(k)}, z_{(k)}\right)} \quad \text { for } k=1, K
$$

The domain is discretized with $K=101 \times 101$ points with a grid spacing of $\Delta=20 \mathrm{~m}$. The constraint condition $T\left(\mathbf{x}_{s}\right)=0$ is enforced as

$$
\sum_{j=1}^{N_{(k)}} T_{(j)} g_{1}^{00}\left(\xi_{1(k)(j)}, \xi_{2(k)(j)}\right) A_{(j)}=0 \text { for }\left(x_{(k)}=x_{s}, z_{(k)}=z_{s}\right)
$$

The initial guess for traveltime is assumed in the form

$$
T_{\text {initial }}(\mathbf{x})=\frac{(x-1)^{2}+(z-1)^{2}}{2}
$$

Fig. 7 compares the PD solution with those of analytical and FMM solutions. The PD solution is achieved when $\|\mathbf{F}(\mathbf{u})\|<6.86 \times 10^{-9}$. The absolute error comparisons of the PD and $1^{\text {st }}$ order FMM solutions against the analytical solution are shown in Fig. 8. The PD error shown in Fig. 8a is relatively uniform and smaller than that for the FMM shown in Fig. 8b. Maximum error occurs at the constraint location due to the enforcement of zero traveltime. Excluding the constraint location, the error in the PD solution is consistently one order of magnitude smaller than the error associated with FMM. The PD prediction certainly agrees with the analytical solution promising superior accuracy even though the curvature of the traveltime near the source location is 
extremely large. Also, it is worth noting that the PD solution is constructed based on the unfactorized form of the Eikonal equation.
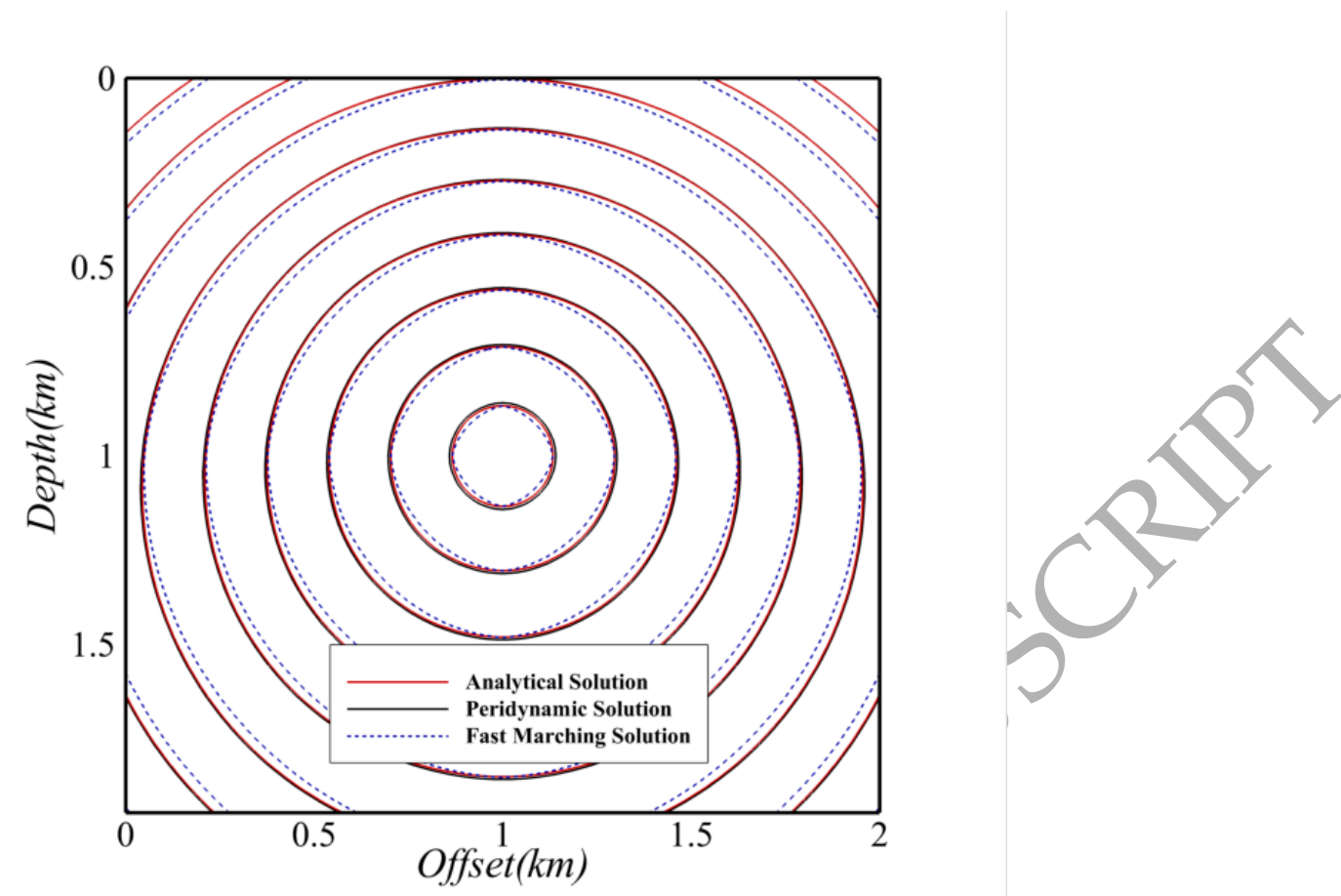

Figure 7. Comparison of PD traveltime predictions with the analytical and FM solutions -

Isotropic Eikonal equation with constant velocity gradients 


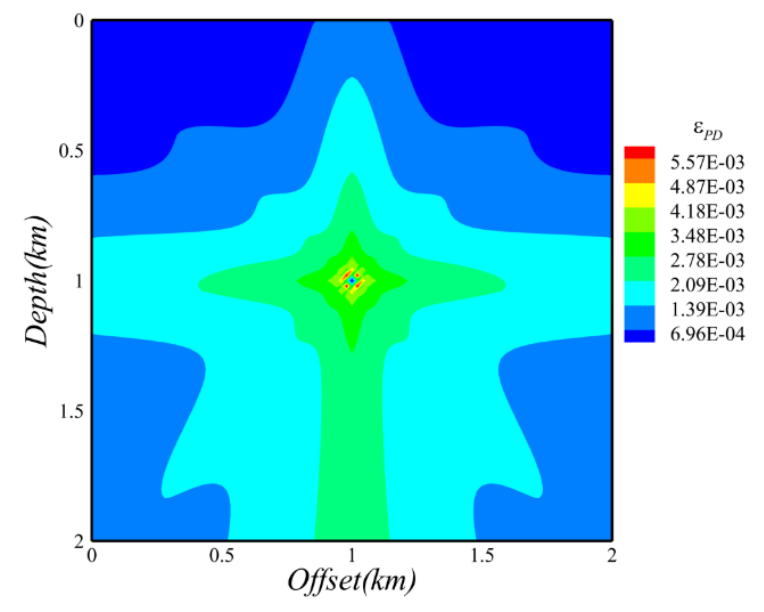

(a)

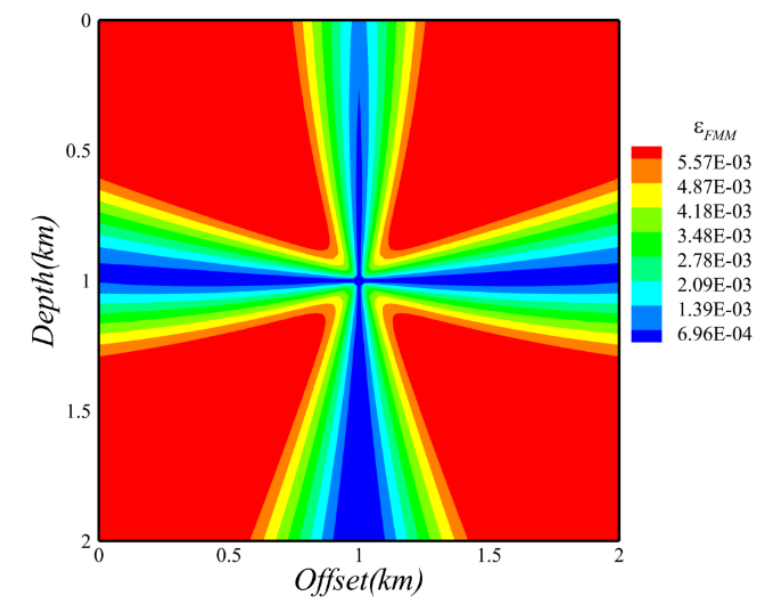

(b)

Figure 8. Absolute error against the analytical solution: a) PD, and ) FMM - Isotropic Eikonal equation with constant velocity gradients

\subsubsection{Marmousi velocity model}

Substituting for the local derivatives from Eq. (39) in Eq. (21) results in the discrete form of the isotropic Eikonal equation as

$$
\left(\sum_{j=1}^{N_{(k)}} T_{(j)} g_{1}^{10}\left(\xi_{1(k)(j)}, \xi_{2(k)(j)}\right) A_{(j)}\right)^{2}+\left(\sum_{j=1}^{N_{(k)}} T_{(j)} g_{1}^{01}\left(\xi_{1(k)(j)}, \xi_{2(k)(j)}\right) A_{(j)}\right)^{2}=\frac{1}{v^{2}\left(x_{(k)}, z_{(k)}\right)} \quad \text { for } k=1, K
$$

with $x \in[0,3 \cdot 0 \mathrm{~km}]$ and $z \in[0,3 \cdot 0 \mathrm{~km}]$. Considering the coarse Marmousi velocity model with a source at $\mathbf{x}_{s}=(1.5 \mathrm{~km}, 1.5 \mathrm{~km})$ shown in Fig. 9., the domain is discretized with $K=101 \times 101$ 
points. The corresponding grid spacing is $\Delta=30 \mathrm{~m}$. The constraint condition, $T\left(\mathbf{x}_{s}\right)=0$ is enforced as

$\sum_{j=1}^{N_{(k)}} T_{(j)} g_{1}^{00}\left(\xi_{1(k)(j)}, \xi_{2(k)(j)}\right) A_{(j)}=0$ for $\left(x_{(k)}=x_{s}, z_{(k)}=z_{s}\right)$

The initial guess for the smoothed velocity field is constructed with the average velocity gradients $\bar{g}_{x}=0$ and $\bar{g}_{z}=1.5 \mathrm{~s}^{-1}$. With $\bar{v}\left(\mathbf{x}_{s}\right)=3.75 \mathrm{~km} / \mathrm{s}$, the initial guess for traveltime becomes

$T_{\text {initial }}(\mathbf{x})=\frac{1}{1.5} \cosh ^{-1}\left(1+\frac{1.5^{2}|\mathbf{x}-(1.0,1.0)|^{2}}{7.5 \bar{v}(\mathbf{x})}\right)$

The solution of the smoothed velocity field becomes the initial guess for the coarse velocity field. Fig. 10 compares the PD predictions solution with those of reference solution and FSM. It is clear that the PD prediction is closer to the reference solution than that of FSM. The PD solution is achieved when $\|\mathbf{F}(\mathbf{u})\|<9 \times 10^{-3}$ and the error comparison against the reference solution is shown in Fig. 11. The error is relatively uniform and smaller than that of FSM. 


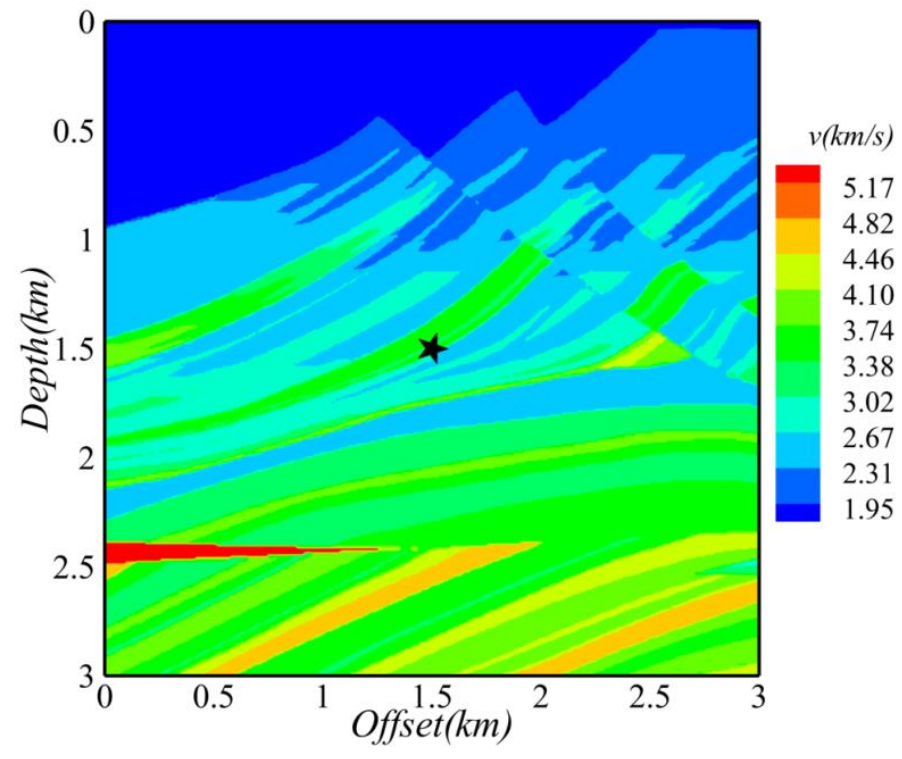

Figure 10. Comparison of PD traveltime predictions with the reference and FSM - Isotropic Eikonal equation 


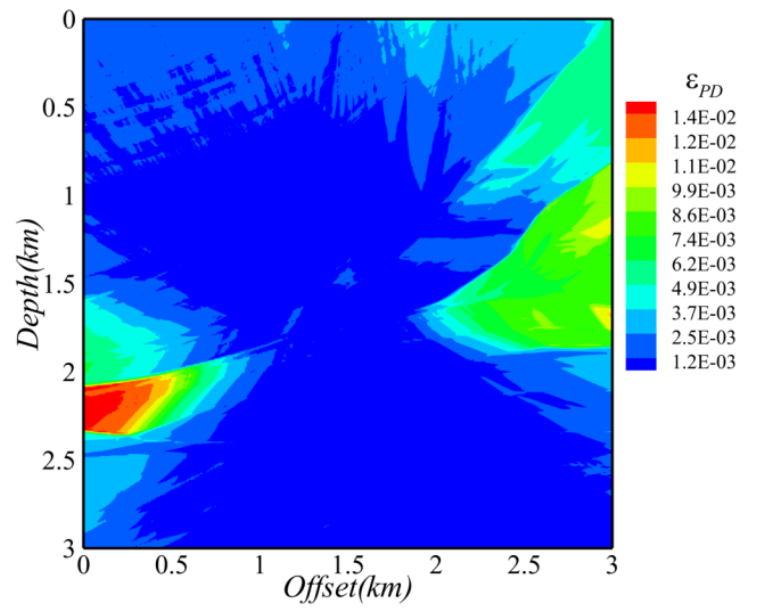

(a)

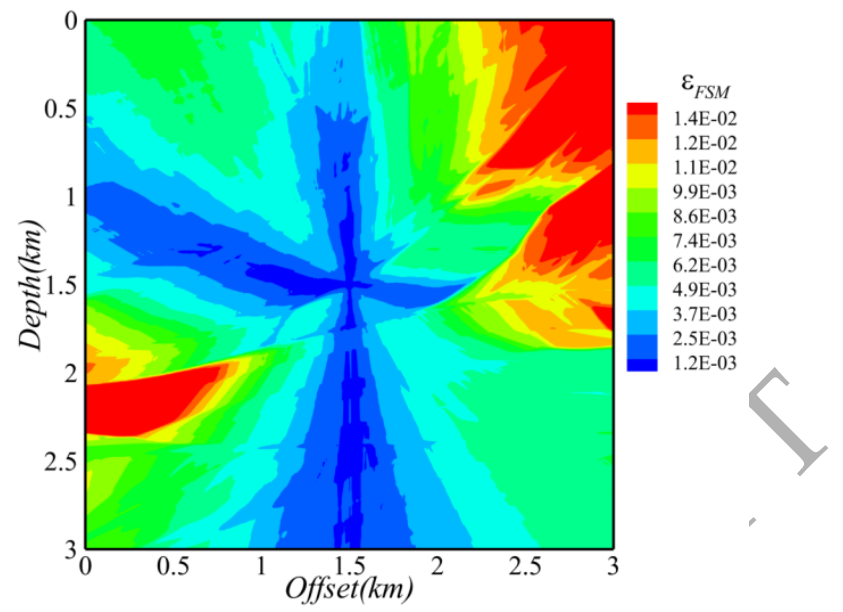

(b)

Figure 11. Absolute error against reference solution: a) PD, and ) FSM - Isotropic Eikonal

\subsection{BP TTI Model}

equation

Substituting for the local derivatives from Eq. (39) in Eq. (19) results in the discrete form of the VTI Eikonal equation as 


$$
\begin{aligned}
& \left(1+2 \varepsilon\left(x_{(k)}, z_{(k)}\right)\right)\left(\begin{array}{l}
\left(\sum_{j=1}^{N_{(k)}} T_{(j)} g_{1}^{10}\left(\xi_{1(k)(j)}, \xi_{2(k)(j)}\right) A_{(j)}\right) \cos \left(\theta\left(x_{(k)}, z_{(k)}\right)\right) \\
+\left(\sum_{j=1}^{N_{(k)}} T_{(j)} g_{1}^{01}\left(\xi_{1(k)(j)}, \xi_{2(k)(j)}\right) A_{(j)}\right) \sin \left(\theta\left(x_{(k)}, z_{(k)}\right)\right)
\end{array}\right)^{2} \text { for } k=1, K \\
& +\left(\begin{array}{l}
\left(\sum_{j=1}^{N_{(k)}} T_{(j)} g_{1}^{01}\left(\xi_{1(k)(j)}, \xi_{2(k)(j)}\right) A_{(j)}\right) \cos \left(\theta\left(x_{(k)}, z_{(k)}\right)\right) \\
-\left(\sum_{j=1}^{N_{(k)}} T_{(j)} g_{1}^{10}\left(\xi_{1(k)(j)}, \xi_{2(k)(j)}\right) A_{(j)}\right) \sin \left(\theta\left(x_{(k)}, z_{(k)}\right)\right)
\end{array}\right) \\
& \times\left(\begin{array}{l}
\left(\sum_{j=1}^{N_{(k)}} T_{(j)} g_{1}^{10}\left(\xi_{1(k)(j)}, \xi_{2(k)(j)}\right) A_{(j)}\right) \cos \left(\theta\left(x_{(k)}, z_{(k)}\right)\right) \\
\left(-R\left(x_{(k)}, z_{(k)}\right)\left(\begin{array}{l}
N_{(k)} \\
+\left(\sum_{j=1} T_{(j)} g_{1}^{01}\left(\xi_{1(k)(j)}, \xi_{2(k)(j)}\right) A_{(j)}\right) \sin \left(\theta\left(x_{(k)}, z_{(k)}\right)\right)
\end{array}\right)^{2}\right)
\end{array}\right)
\end{aligned}
$$

with $x \in[0,10 \mathrm{~km}]$ and $z \in[0,10 \mathrm{~km}]$. The velocity field shown in Fig. 12 has a source at $\mathbf{x}_{s}=(5 \mathrm{~km}, 0.5 \mathrm{~km})$. The variation of anisotropy parameters, $\varepsilon(x, z)$ and $\eta(x, z)$ and tilt angle, $\theta(x, z)$ are shown in Figs. 13, 14 and 15, respectively. Although the topography at the surface is irregular, the domain is discretized with $K=161 \times 161$ points. The corresponding uniform grid spacing is $\Delta=62.5 \mathrm{~m}$. The constraint condition, $T\left(\mathbf{x}_{s}\right)=0$ is enforced as

$$
\sum_{j=1}^{N_{(k)}} T_{(j)} g_{1}^{00}\left(\xi_{1(k)(j)}, \xi_{2(k)(j)}\right) A_{(j)}=0 \text { for }\left(x_{(k)}=x_{s}, z_{(k)}=z_{s}\right)
$$

The initial guess for the traveltime field is constructed with the average velocity gradients $\bar{g}_{x}=0$ and $\bar{g}_{\bar{z}}=0.35 \mathrm{~s}^{-1}$. With $\bar{v}\left(\mathbf{x}_{s}\right)=1.175 \mathrm{~km} / \mathrm{s}$, the initial guess for traveltime becomes 
$T_{\text {initial }}(\mathbf{x})=\frac{1}{0.35} \cosh ^{-1}\left(1+\frac{0.35^{2}|\mathbf{x}-(5,5)|^{2}}{2.35 \bar{v}(\mathbf{x})}\right)$

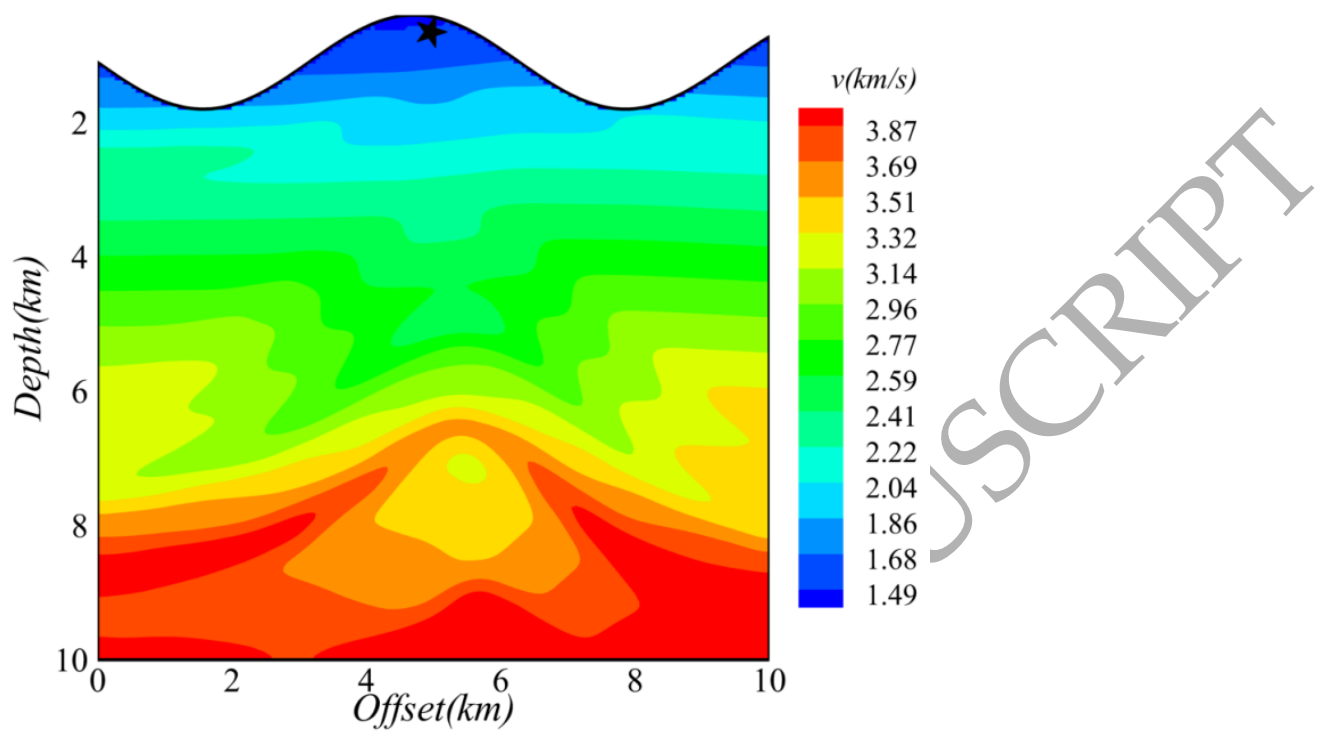

Figure 12. Velocity field, $v(x, z)$ in the domain with a source at $\mathbf{x}_{s}=(5.0,0.5)$ - BP TTI model 


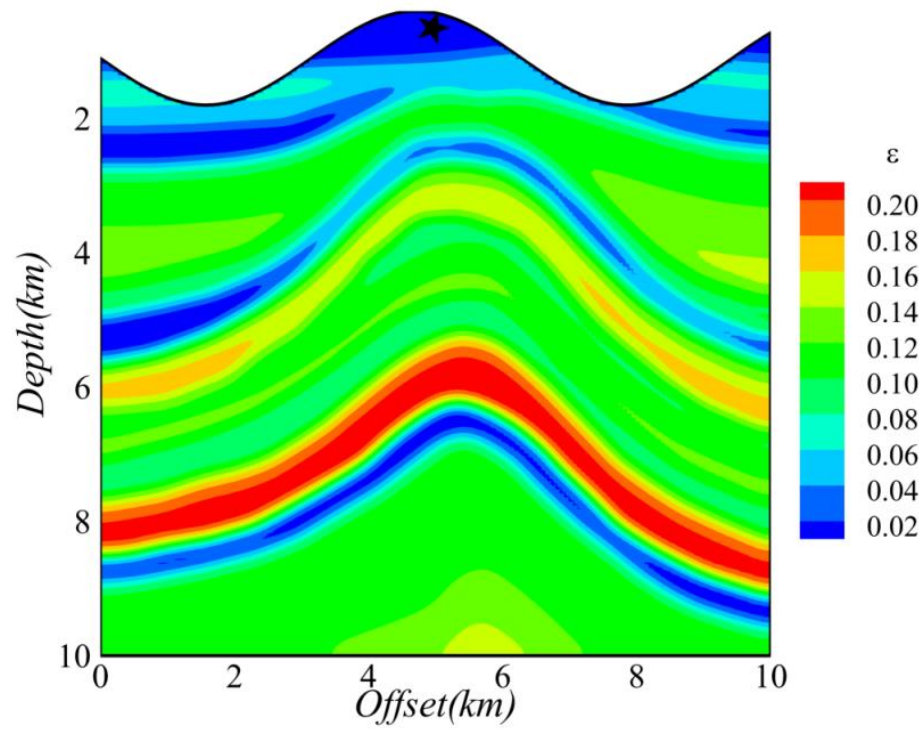

Figure 14. Variation of anisotropy parameter $\eta(x, z)$ in the domain - BP TTI model 


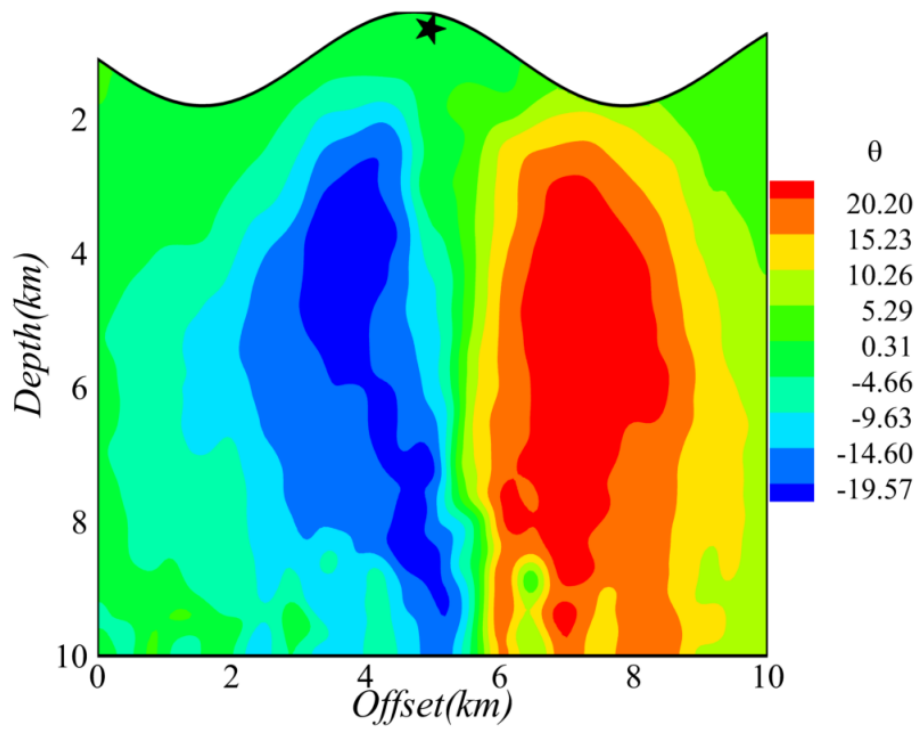

Fig. 16 compares the PD prediction with those of reference solution and FSM. The PD solution is closer to the reference solution than that of FSM. The PD solution is achieved when $\|\mathbf{F}(\mathbf{u})\|<3.17 \times 10^{-3}$ and the error comparison against the reference solution is shown in Fig. 17. The error is relatively uniform and smaller than that of FSM as shown in Fig. 18. 


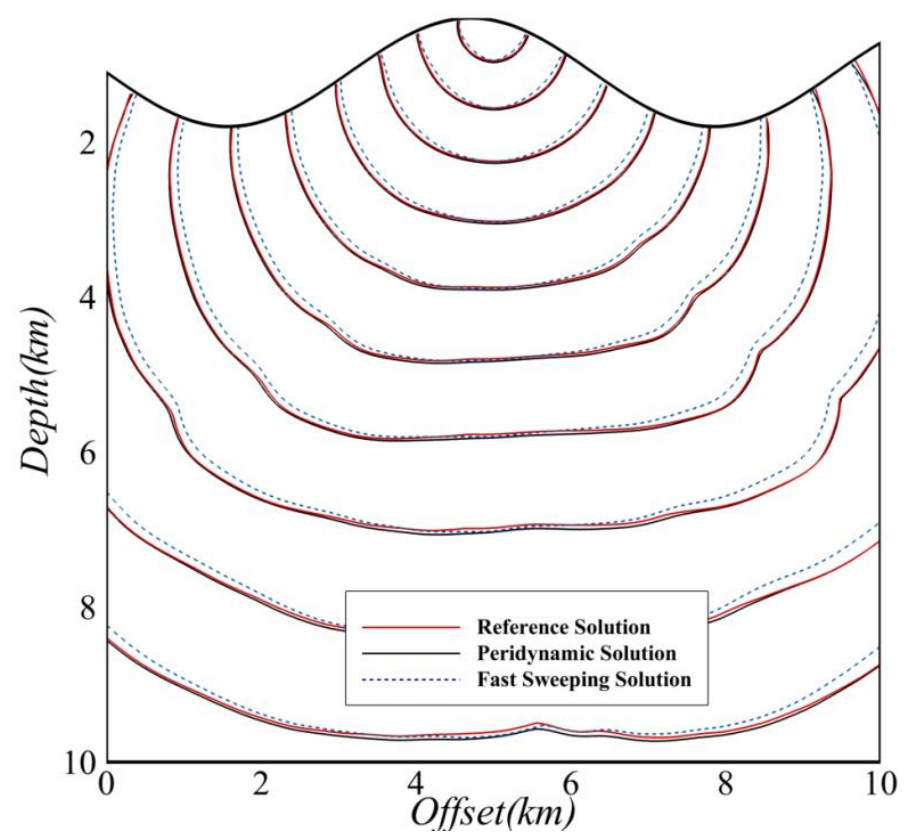

Figure 17. Absolute error measure in PD solution against reference solution - BP TTI model 


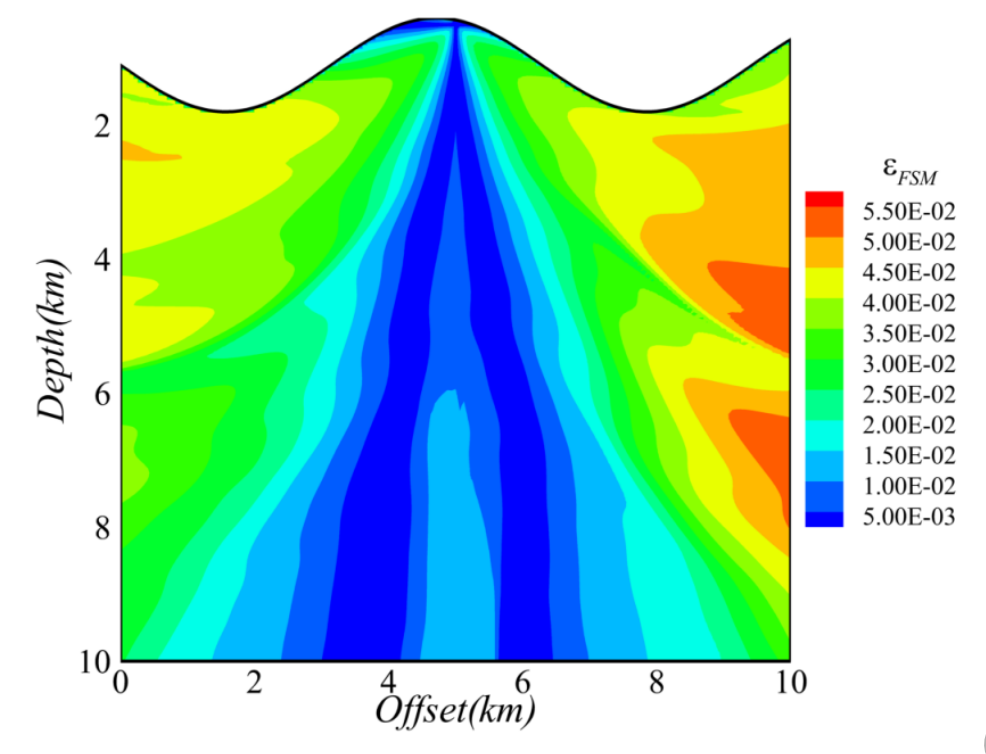

\section{$5.3 \mathrm{qSV}$-wave}

Substituting for the local derivatives from Eq. (39) in Eq. (25) results in the discrete form of the qSV wave VTI Eikonal equation as

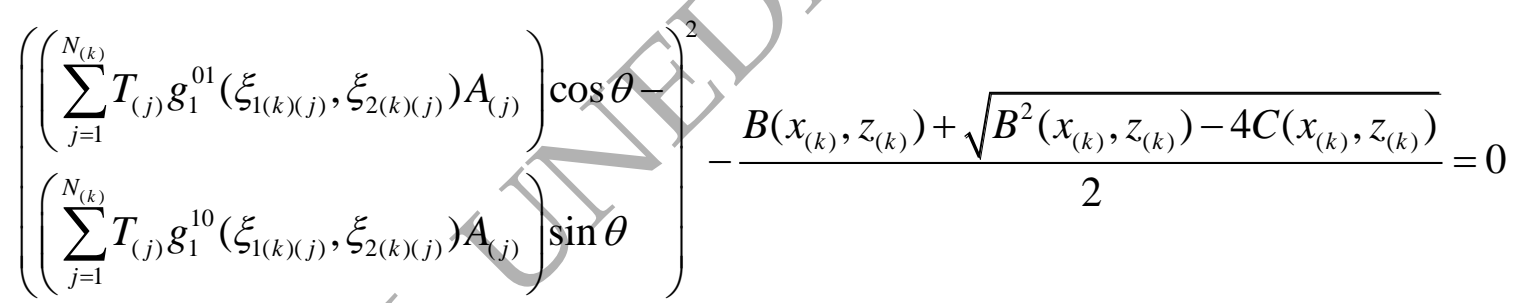

in which 


$$
B\left(x_{(k)}, z_{(k)}\right)=\frac{1}{v_{p}^{2}}+\frac{1}{v_{s}^{2}}-2\left(1+\delta+(\varepsilon-\delta) \frac{v_{p}^{2}}{v_{s}^{2}}\right)\left(\begin{array}{l}
\left(\sum_{j=1}^{N_{(k)}} T_{(j)} g_{1}^{10}\left(\xi_{1(k)(j)}, \xi_{2(k)(j)}\right) A_{(j)}\right) \cos \theta \\
\left.+\left(\sum_{j=1}^{N_{(k)}} T_{(j)} g_{1}^{01}\left(\xi_{1(k)(j)}, \xi_{2(k)(j)}\right) A_{(j)}\right) \sin \theta\right)^{2}
\end{array}\right.
$$

and

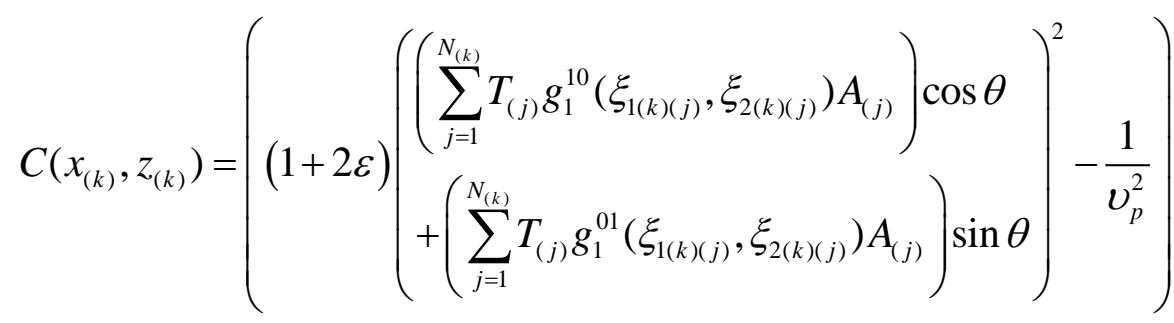

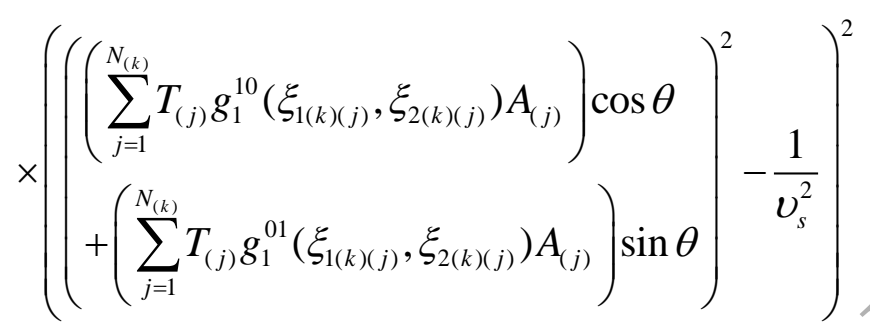

with $x \in[0,2 \mathrm{~km}]$ and $z \in[0,2 \mathrm{~km}]$. The $\mathrm{P}$ - and $\mathrm{S}$-wave phase velocity fields with constant gradients are shown in Fig. 19 with a source at $\mathbf{x}_{s}=(1.0 \mathrm{~km}, 1.0 \mathrm{~km})$. The Thomsen anisotropy parameters have uniform values of $\epsilon=0.4$ and $\delta=0.2$, and the homogeneous tilt angle is $\theta=40^{\circ}$. the domain is discretized with $K=101 \times 101$ points. The corresponding uniform grid spacing is $\Delta=10 \mathrm{~m}$. The constraint condition, $T\left(\mathbf{x}_{s}\right)=0$ is enforced as

$\sum_{j=1}^{N_{(k)}} T_{(j)} g_{1}^{90}\left(\xi_{(k)(j)}, \xi_{2(k)(j)}\right) A_{(j)}=0$ for $\left(x_{(k)}=x_{s}, z_{(k)}=z_{s}\right)$ 
The initial guess for the traveltime field is constructed with the average velocity gradients $\bar{g}_{x}=0$ and $\bar{g}_{z}=0.5 s^{-1}$. With $v_{p}\left(\mathbf{x}_{s}\right)=2.5 \mathrm{~km} / \mathrm{s}$, the initial guess for traveltime becomes

$T_{\text {initial }}(\mathbf{x})=2 \cosh ^{-1}\left(1+\frac{|\mathbf{x}-(1.0,1.0)|^{2}}{20 v(\mathbf{x})}\right)$

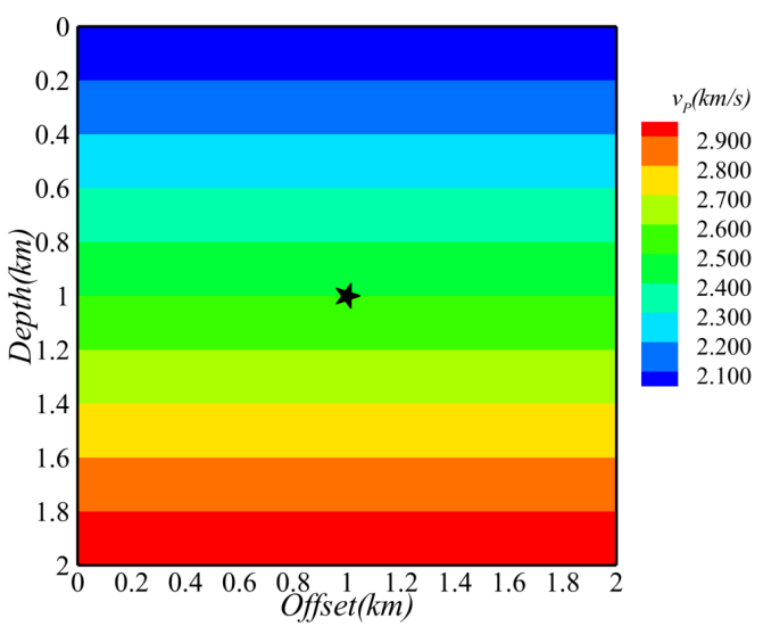

(a)

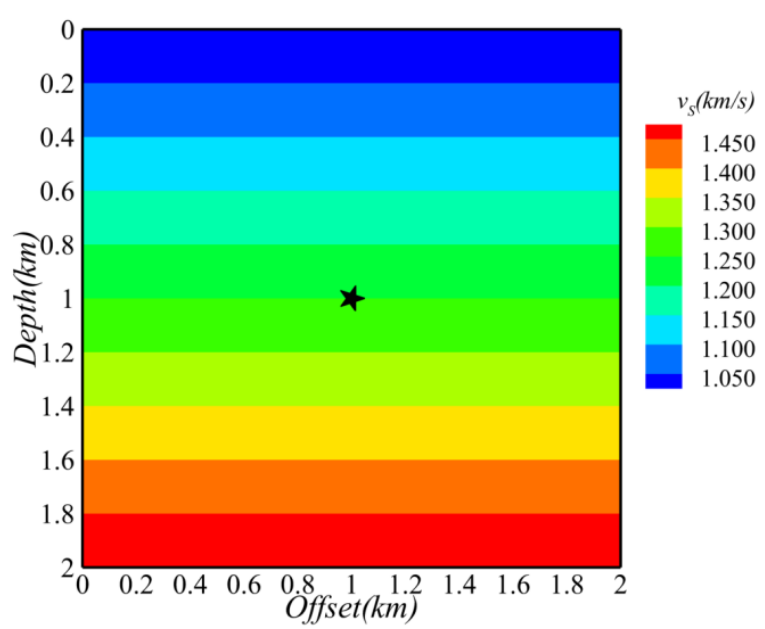

(b)

Figure 19. Velocity field in the domain with a source at $\mathbf{x}_{s}=(1.0,1.0)$ : a) P-, and b) S-wave

Fig. 20 compares the PD prediction with that of low-rank approximation at $t=0.63 \mathrm{~s}$ (Fomel et al. 2013). The PD solution is achieved when $\|\mathbf{F}(\mathbf{u})\|<1.877 \times 10^{-13}$. The PD solution recovers the main branch of the solution due its nonlocality and numerical dissipation. 


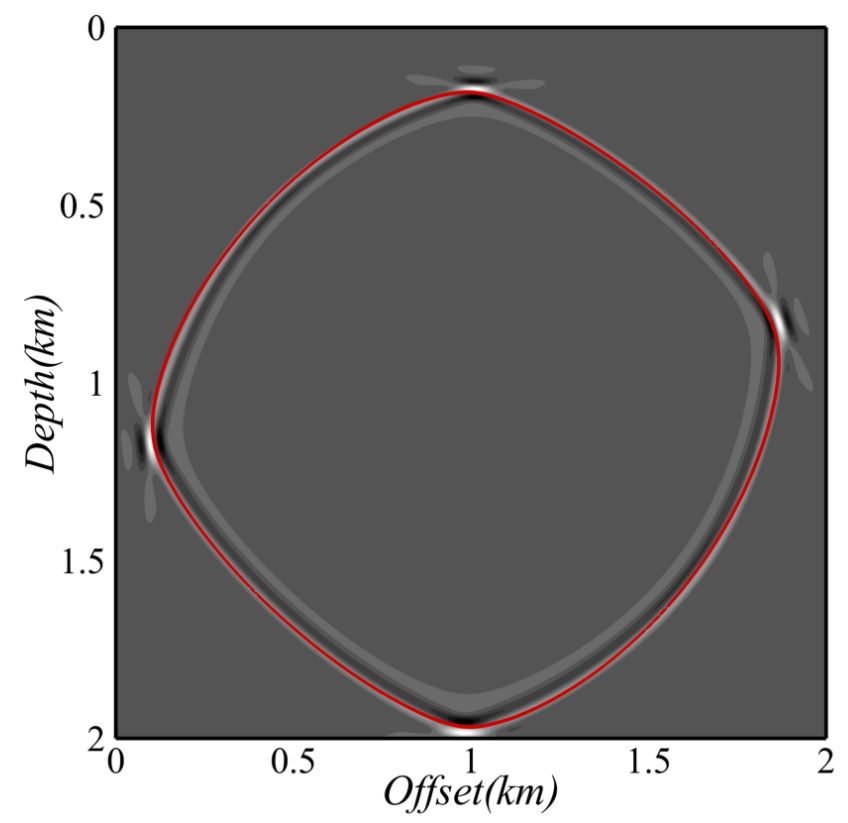

Figure 20. Comparison of PD prediction of traveltime contour (red) with the low-rank solution at

\section{CONCLUSIONS}

$t=0.63 s$

The study presents a new approach for solving different types of the Eikonal equation by using the nonlocal PDDO. The PDDO provides the nonlocal form of Eikonal equations with directional nonlocality. The PDDO solution handles discontinuities very well and invokes the direction of information travel in a consistent manner. The weight function controls the strength of association among the family members. Also, it enables directional nonlocality based on the knowledge of characteristic directions along which information travels. Solutions are constructed in a consistent manner without any special treatments through simple discretization. The construction of the initial guess for the traveltime is systematic and automatic differentiation is used to calculate the Jacobian matrix at every iteration. 
The PDDO successfully provides accurate solutions to different types of challenging Eikonal equations with complex velocity fields in complex anisotropic media and with irregular topography. Unlike FSM or FMM, the PDDO does not require any modification. Tracking the gradients of traveltimes is adequate to capture both the group and phase velocity vectors; the results are stable and accurate. Although not investigated, the computational time may be more costly than the FSM and FMM. However, the PDDO is extremely suitable for parallelization, particularly on GPU (Graphics Processing Unit) using CUDA software. This feature can be explored for computational speed up especially when considering three-dimensional Eikonal equations.

\section{ACKNOWLEDGEMENT}

This study was performed as part of the ongoing research at the MURICenter for Material Failure Prediction through Peridynamics at the University of Arizona (AFOSR Grant No. FA9550-14-10073).

\section{CODE AVAILABILITY}

The software code underlying this study will be shared on reasonable request to the corresponding author.

\section{REFERENCES}

Adalsteinsson, D. \& Sethian, J.A., 1995. A Fast Level Set Method for Propagating Interfaces, J. Comput. Phys., 118, 269-277. 
Alkhalifah, T., 1998. Acoustic approximations for processing in transversely isotropic media, Geophysics, 63, 623-631.

Alkhalifah, T., 2000. An acoustic wave equation for anisotropic media, Geophysics, 65, 12391250

Alkhalifah, T., 2003, An acoustic wave equation for orthorhombic anisotropy, Geophysics, 68, 1169-1172.

Alvino, C., Unal, G., Slabaugh, G., Peny, B. \& Fang, T., 2007. Efficient segmentation based on Eikonal and diffusion equations, Int. J. Comput. Math., 84, 1309-1324.

Bekar, A.C. \& Madenci, E., 2021. Peridynamics enabled learning partial differential equations, $J$. Comput. Phys., 434, 110193.

Bentley, J.L., 1975. Multidimensional binary search trees used for associative searching, Communications of the ACM 18, 18, 509-517.

Boys, B., Dodwell, T. J., Hobbs, M., \& Girolami, M., 2021, PeriPy--A High Performance OpenCL Peridynamics Package, Comput. Methods Appl. Mech. Eng., 386, 114085.

Bullen, K.E., 1937. Seismic Ray Theory Vol. 4, Cambridge University Press.

Cao, Z., Pan, S., Li, R., Balachandran, R., Fitzpatrick, J.M., Chapman, W.C. \& Dawant, B.M., 2004. Registration of medicalimages using an interpolated closest point transform: Method and validation, Med. Image Anal., 8, 421-427

Detrixhe, M. \& Gibou, F., 2016. Hybrid massively parallel fast sweeping method for static Hamilton-Jacobi equations, J. Comput. Phys., 322, 199-223.

Dijkstra, E.W., 1959. A note on two problems in connexion with graphs, Numer. Math., 1, 269271 .

Eringen, A.C., 2002. Nonlocal continuum field theories, Springer, New York, NY. 
Fomel, S., Luo, S. \& Zhao, H., 2009. Fast sweeping method for the factored eikonal equation, J. Comput. Phys., 228, 6440-6455

Fomel, S., Ying, L. \& Song, X.,2013. Seismic wave extrapolation using lowrank symbol approximation, Geophys. Prospect., 61, 526-536.

Garrido, S., Álvarez, D. \& Moreno, L., 2016. Path planning for mars rovers using the fast marching method, Advances in Intelligent Systems and Computing, 417, 93-105.

Grechka, V., De La Pena, A., Schisselé-Rebel, E., Auger, E. \& Roux, P.F., 2015. Relative location of microseismicity, Geophysics, 80, WC1-WC9.

Han, S., Zhang, W. \& Zhang, J., 2017. Calculating qP-wave traveltimes in 2-D TTy media by highorderfast sweeping methods with a numerical quartic equation solver, Geophys. J. Int., 210, 1560-1569.

Helmsen, J.J., Puckett, E.G., Colella, P. \& Dorr, M., 1996. Two new methods for simulating photolithography development in 3D, Optical Microlithography IX, 2726, 253-261.

Lambare, G., Operto, S., Podvin, P. \& Thierry, P., 2003. 3D ray+ Born migration/inversion-Part 1: Theory, Geophysics, 68, 1126-1422.

Lan, H. \& Zhang, Z., 2013. Topography-dependent eikonal equation and its solver for calculating first-arrival traveltimes with an irregular surface, Geophys. J. Int., 193, 1010-1026.

Luo, S. \& Qian, J., 2012. Fast sweeping methods for factored anisotropic eikonal equations: Multiplicative and additive factors, J. Sci. Comput., 52, 360-382.

Madenci, E., Barut, A. \& Futch, M., 2016. Peridynamic differential operator and its applications, Computer Methods in Applied Mechanics and Engineering, 304, 408-451. 
Madenci, E., Barut, A., Dorduncu, M. \& Futch, M., 2017. Numerical solution of linear and nonlinear partial differential equations by using the peridynamic differential operator, Numerical Methods for Partial Differential Equations, 33, 1726-1753.

Madenci, E., Barut, A. \& Dorduncu, M., 2019. Peridynamic differential operators for numerical analysis, Springer, Boston, MA.

Malladi, R. \& Sethian, J.A., 1996. A unified approach to noise removal, image enhancement, and shape recovery, IEEE Trans. Image Process., 5, 1554-1568.

Musgrave, M. J. P., 1970, Crystal Acoustics, Holden - Day, San Francisco.

Popovici, A.M. \& Sethian, J.A., 2002. 3-D imaging using higher order fast marching traveltimes, Geophysics, 67, 604-609.

Qin, F., Luo, Y., Olsen, K.B., Cai, W. \& Schuster, G.T., 1992. Finite-difference solution of the eikonal equation along expanding wavefronts, Geophysics, 57, 478-487.

Raviv, D., Bronstein, A.M., Bronstein, M.M., Kimmel, R. \& Sochen, N., 2011. Affine-invariant geodesic geometry of deformable 3D shapes, Comput. Graph., 35, 692-697.

Sethian, J.A., 1996. A fast marching level set method for monotonically advancing fronts, Proc. Natl. Acad. Sci. U. S. A., 93, 1591-1595.

Silling, S.A., 2000. Reformulation of elasticity theory for discontinuities and long-range forces, $J$. Mech. Phys. Solids, 48, 175-209.

Sharma, M. D., 2008, Propagation of inhomogeneous plane waves in anisotropic viscoelastic media, Acta mechanica, 200, 145-154.

Taillandier, C., Noble, M., Chauris, H. \& Calandra, H., 2009. First-arrival traveltime tomography based on the adjoint-state method, Geophysics, 74, WCB1-WCB10. 
Tsitsiklis, J.N., 1995. Efficient Algorithms for Globally Optimal Trajectories, IEEE Trans. Automat. Contr., 40, 1528-1538.

Vavryčuk, V., 2010, Behaviour of rays at interfaces in anisotropic viscoelastic media, Geophys. J. Int., 181, 1665-1677

Ventura, R. \& Ahmad, A., 2015. Towards optimal robot navigation in domestic spaces, Lecture Notes in Artificial Intelligence, 8992, 318-331.

Vidale, J.E., 1989. Finite-difference calculation of traveltimes in 3-D, Geophysics, 55, 521-526.

Waheed, U. B., Yarman, C. E., \& Flagg, G., 2015, An iterative, fast-sweeping-based eikonal solver for 3D tilted anisotropic media, Geophysics, 80, C49-C58.

Waheed, U.b. \& Alkhalifah, T., 2017. A fast sweeping algorithm for accurate solution of the tilted transversely isotropic eikonal equation using factorization, Geophysics, 82, WB1-WB8

Waheed, U.b., Alkhalifah, T., Haghighat, E. \& Song, C., 2021. A hølistic approach to computing first-arrival traveltimes using neural networks, arXiv: arXiv:2101.11840

Zhang, Y.T. \& Qian, J., 2004. High order fast sweeping methods for Eikonal equations, 2004 SEG Аnnu. Meet., 29, 25-56.

Zhao, H., 2005. A Fast Sweeping Method for Eikonal Equations, Math. Comput., 74, 603-627. 


\section{APPENDIX}

Solutions to the elliptically isotropic Marmousi model and the Hess VTI model are presented in order to expose the generality of the PDDO while retaining focus and coherence.

\section{A.1 Elliptic Eikonal equation}

With the PD representation of the local derivatives from Eq. (39), the discrete form of the Elliptic Eikonal equation, Eq. (20) can be written as

$$
\begin{aligned}
\left(1+2 \varepsilon\left(x_{(k)}, z_{(k)}\right)\right) & \left(\sum_{j=1}^{N_{(k)}} T_{(j)} g_{1}^{10}\left(\xi_{1(k)(j)}, \xi_{2(k)(j)}\right) A_{(j)}\right)^{2} \\
& +\left(\sum_{j=1}^{N_{(k)}} T_{(j)} g_{1}^{01}\left(\xi_{1(k)(j)}, \xi_{2(k)(j)}\right) A_{(j)}\right)^{2}=\frac{1}{v^{2}\left(x_{(k)}, z_{(k)}\right)}
\end{aligned}
$$

with $x \in[0,2.9875 \mathrm{~km}]$ and $z \in[0,2.9875 \mathrm{~km}]$. The velocity field shown in Fig. 9 has a source at $\mathbf{x}_{s}=(1.5 \mathrm{~km}, 2.5 \mathrm{~km})$. The variation of anisotropy parameter, $\varepsilon(x, z)$, shown in Fig. A.1 has abrupt changes in the domain. The domain is discretized with $K=240 \times 240$ points. The corresponding grid spacing is $\Delta=12.5 \mathrm{~m}$. The constraint condition, $T\left(\mathbf{x}_{s}\right)=0$ is enforced as

$$
\sum_{j=1}^{N_{(k)}} T_{(j)} g_{1}^{00}\left(\xi_{1(k)(j)}, \hat{s}_{2(k(k)(j)}\right) A_{(j)}=0 \text { for }\left(x_{(k)}=x_{s}, z_{(k)}=z_{s}\right)
$$


The initial guess for the smoothed velocity field is constructed with the average velocity gradients $\bar{g}_{x}=0$ and $\bar{g}_{z}=1.5 s^{-1}$. With $\bar{v}\left(\mathbf{x}_{s}\right)=5.25 \mathrm{~km} / \mathrm{s}$, the initial guess for traveltime becomes

$$
T_{\text {initial }}(\mathbf{x})=\frac{1}{1.5} \cosh ^{-1}\left(1+\frac{1.5^{2}|\mathbf{x}-(1.5,2.5)|^{2}}{10.5 \bar{v}(\mathbf{x})}\right)
$$

The solution to the smoothed velocity field becomes the initial gues for the coarse velocity field.
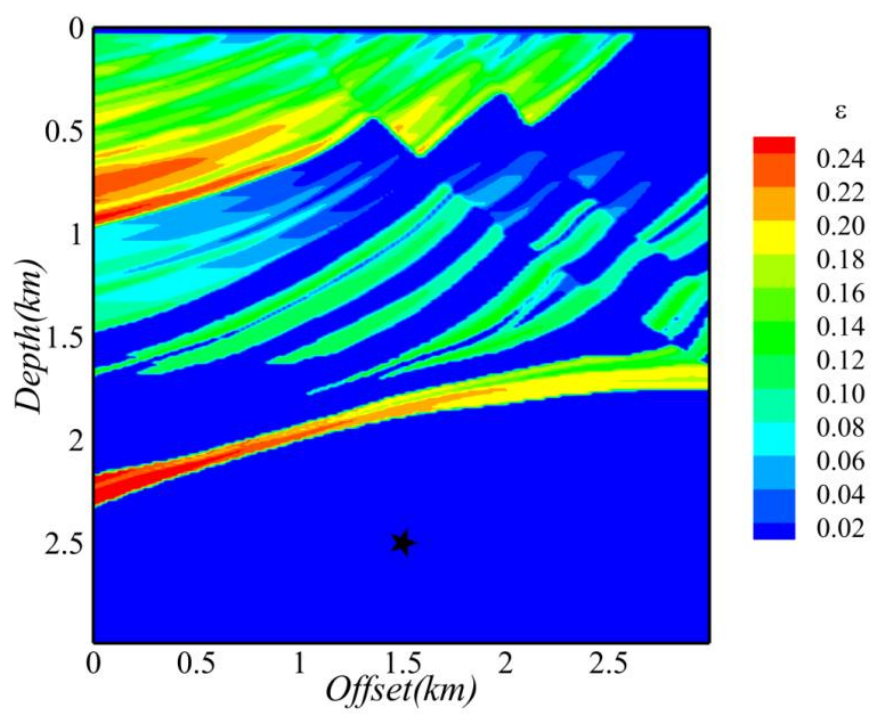

0.02

Figure A.1. Variation of anisotropic parameter $\varepsilon(x, z)$ - Elliptic Eikonal equation

Fig. A.2 compares the PD prediction with those of reference solution and FSM. The PD solution is achieved when $\|\mathbf{F}(\mathbf{u})\|<2.3 \times 10^{-3}$ and the error comparison against the reference solution is shown in Fig. A.3. It obvious that PD performs better than that of FSM. 


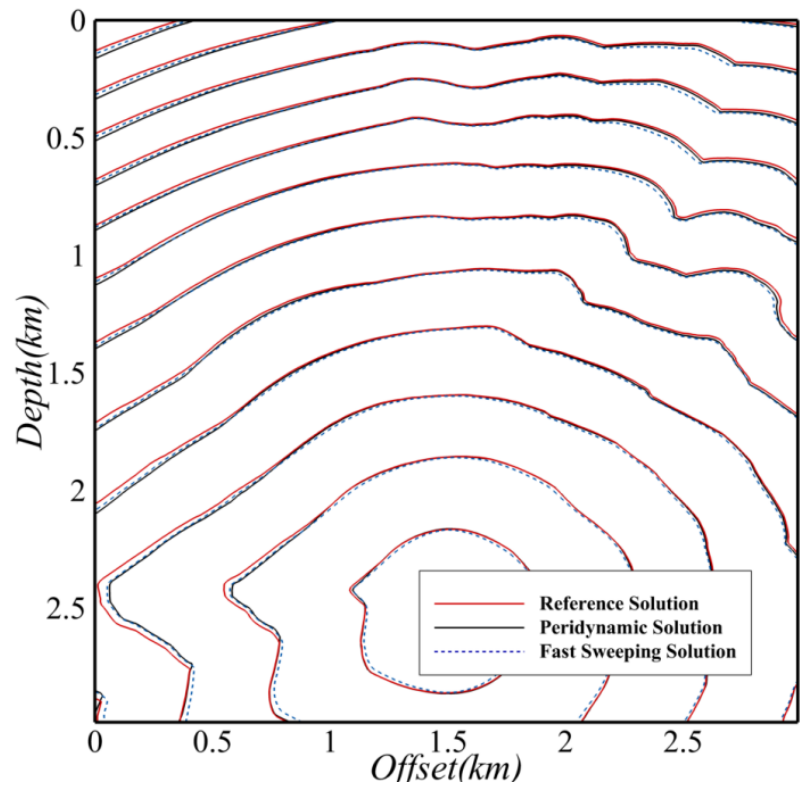

Figure A.2. Comparison of PD traveltime predictions with the referenceand FSM - Elliptic
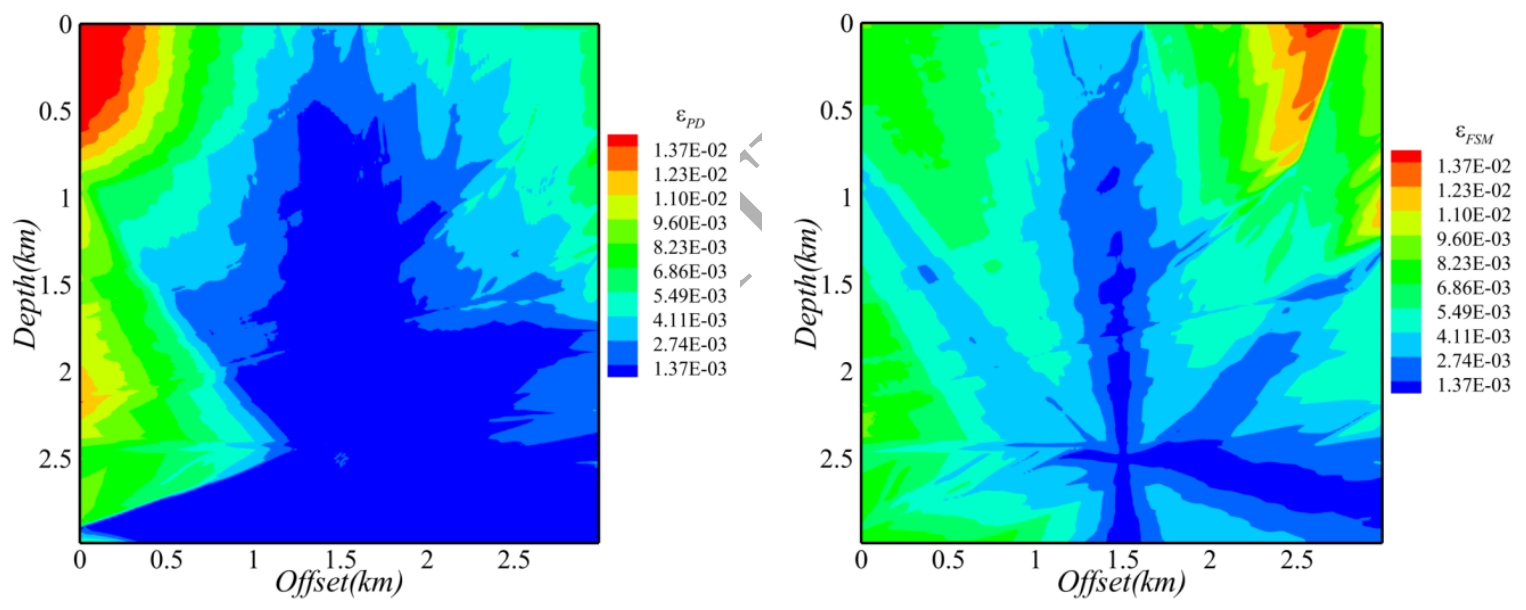

(a)

(b)

Figure A.3. Absolute error against reference solution: a) PD, and b) FSM - Elliptic Eikonal equation 


\section{A.2 Hess VTI Model}

Substituting for the local derivatives from Eq. (39) in Eq. (19) results in the discrete form of the VTI Eikonal equation as

$$
\begin{aligned}
& \left(1+2 \varepsilon\left(x_{(k)}, z_{(k)}\right)\right)\left(\sum_{j=1}^{N_{(k)}} T_{(j)} g_{1}^{10}\left(\xi_{1(k)(j)}, \xi_{2(k)(j)}\right) A_{(j)}\right)^{2}+ \\
& \left(\sum_{j=1}^{N_{(k)}} T_{(j)} g_{1}^{01}\left(\xi_{1(k)(j)}, \xi_{2(k)(j)}\right) A_{(j)}\right)^{2} \times \quad \text { for } k=1, K \\
& \left(1-R\left(x_{(k)}, z_{(k)}\right)\left(\sum_{j=1}^{N_{(k)}} T_{(j)} g_{1}^{10}\left(\xi_{1(k)(j)}, \xi_{2(k)(j)}\right) A_{(j)}\right)^{2}\right)=\frac{1}{v^{2}\left(x_{(k)}, z_{(k)}\right)}
\end{aligned}
$$

with

$$
R\left(x_{(k)}, z_{(k)}\right)=\frac{2 \eta\left(x_{(k)}, z_{(k)}\right) v^{2}\left(x_{(k)}, z_{(k)}\right)\left(1+2 \varepsilon\left(x_{(k)}, z_{(k)}\right)\right)}{1+2 \eta\left(x_{(k)}, z_{(k)}\right)}
$$

with $x \in[0,21.69 \mathrm{~km}]$ and $z \in[0,8.97 \mathrm{~km}]$. The velocity field shown in Fig. A.4 has a source at $\mathbf{x}_{s}=(12 \mathrm{~km}, 3 \mathrm{~km})$. The variation of anisotropy parameters, $\varepsilon(x, z)$ and $\eta(x, z)$ are shown in Figs. A.5 and A.6, respectively. The domain is discretized with $K=724 \times 300$ points. The corresponding grid spacing is $\Delta=30 \mathrm{~m}$. The constraint condition, $T\left(\mathbf{x}_{s}\right)=0$ is enforced as

$\sum_{j=1}^{N_{(k)}} T_{(j)} g_{1}^{00}\left(\xi_{1, k(j)}, \xi_{2(k)(j)}\right) A_{(j)}=0$ for $\left(x_{(k)}=x_{s}, z_{(k)}=z_{s}\right)$ 
The initial guess for the traveltime field is constructed with the average velocity gradients $\bar{g}_{x}=0$ and $\bar{g}_{z}=0.3 s^{-1}$. With $\bar{v}\left(\mathbf{x}_{s}\right)=2.4 \mathrm{~km} / \mathrm{s}$, the initial guess for traveltime becomes

$T_{\text {initial }}(\mathbf{x})=\frac{1}{0.3} \cosh ^{-1}\left(1+\frac{0.3^{2}|\mathbf{x}-(12.0,3.0)|^{2}}{4.8 \bar{v}(\mathbf{x})}\right)$

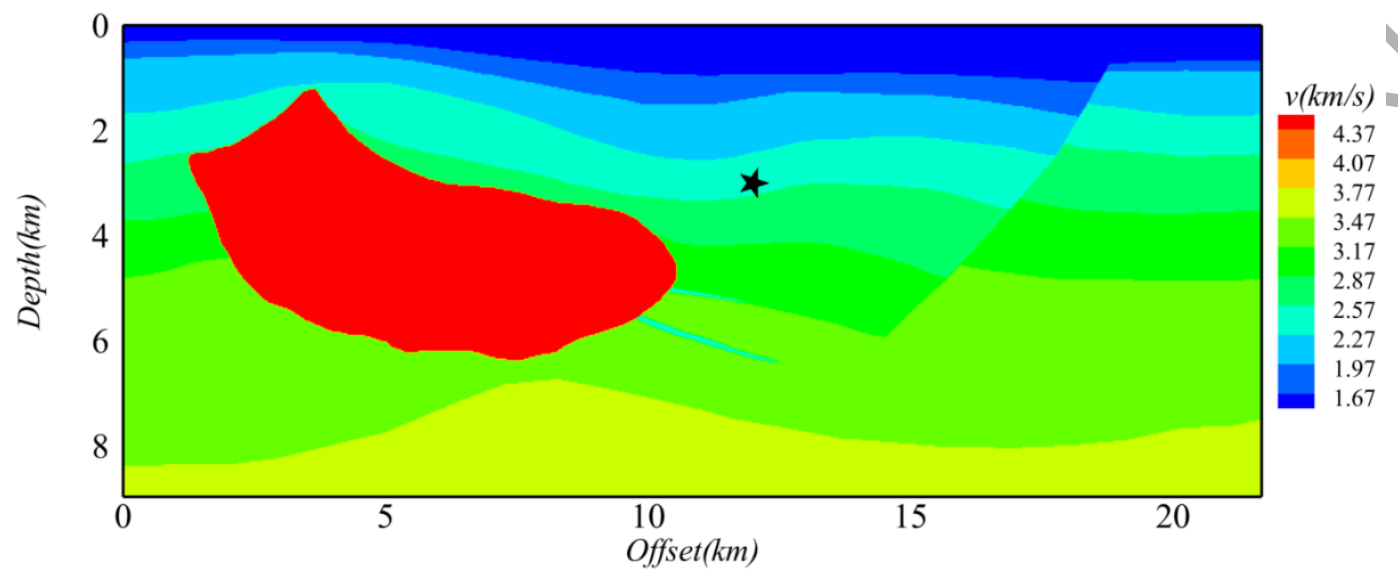

Figure A.4. Velocity field $v(x, z)$ in the domain with a source at $\mathbf{x}_{s}=(12.0,3.0)$ - Hess model

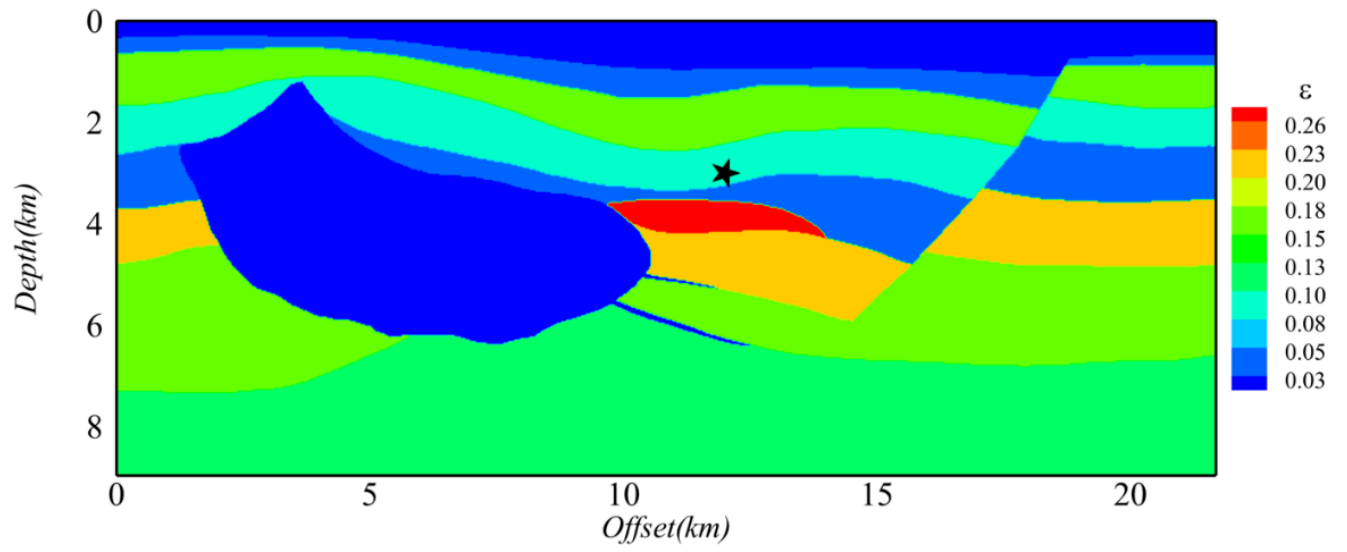

Figure A.5. Variation of anisotropy parameter $\varepsilon(x, z)$ in the domain - Hess model 


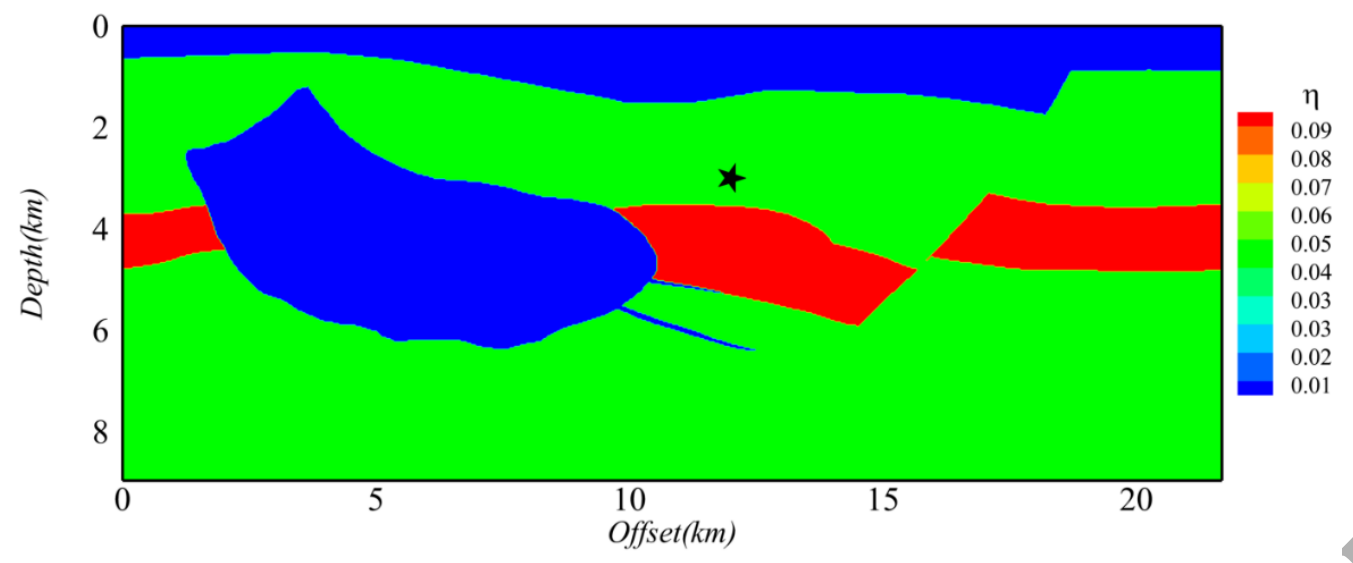

Figure A.6. Variation of anisotropy parameter $\eta(x, z)$ in the domain - Hess model

Fig. A.7 compares the PD prediction with those of reference solution and FSM. The PD solution is closer to the reference solution than that of FSM. The PD solution is achieved when $\|\mathbf{F}(\mathbf{u})\|<1.554 \times 10^{-3}$ and the error comparison against the reference solution is shown in Fig. A.8. The error is relatively uniform and smaller than that of FSM, as shown in Fig. A.9. 


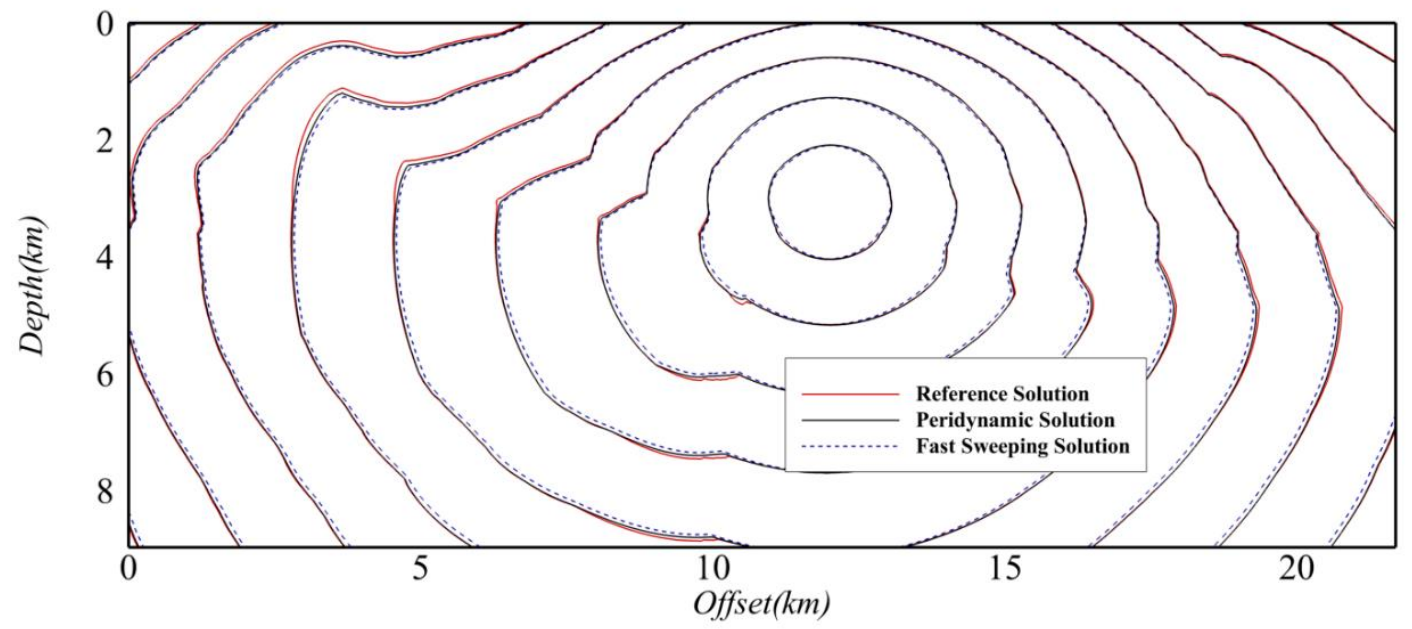

Figure A.8. Absolute error measure in PD solution against reference solution - Hess VTI model 


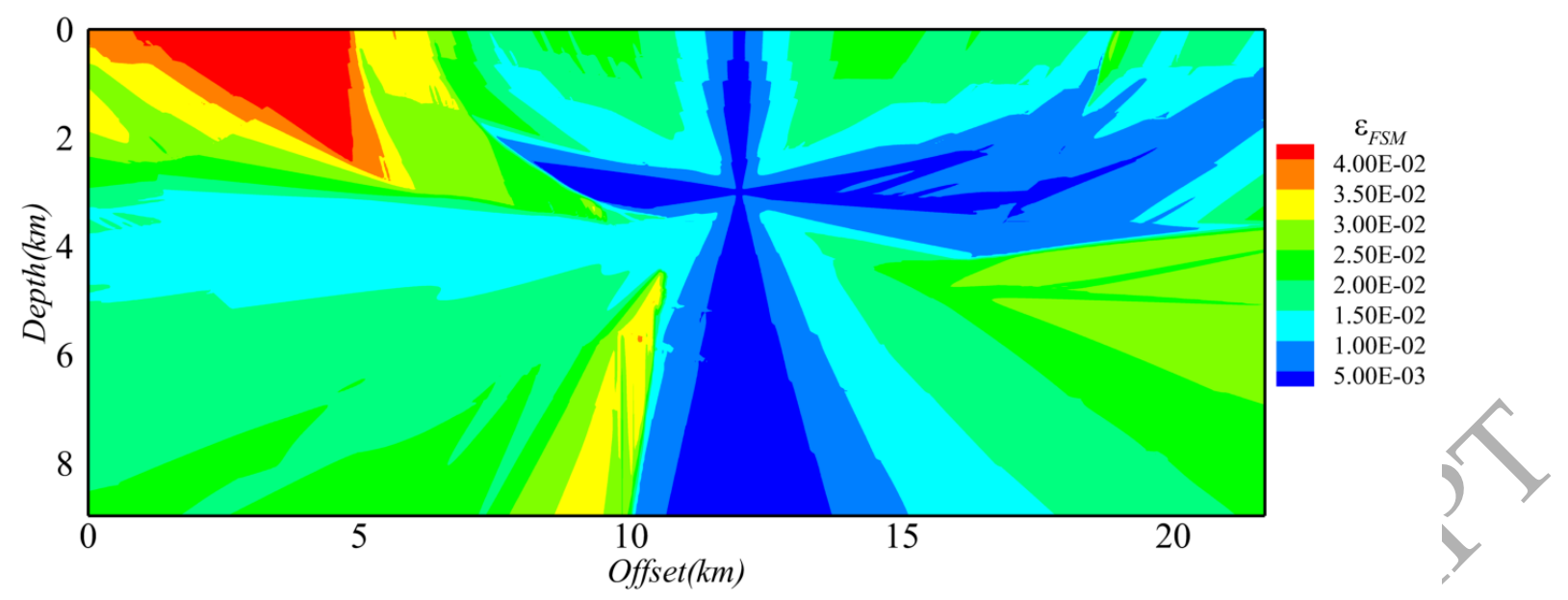

Figure A.9. Absolute error measure in FSM against reference solution - Hess VTI model 